\title{
The dust coma environment of the short period comets 32P/Comas Solá, 56P/Slaughter-Burnham, and 78P/Gehrels 2 from ground-based observations
}

\author{
E. Mazzotta Epifani ${ }^{1}$ and P. Palumbo ${ }^{2}$ \\ 1 INAF - Osservatorio Astronomico di Capodimonte, via Moiariello 16, 80131 Napoli, Italy \\ e-mail: epifani@oacn.inaf.it \\ ${ }^{2}$ Universitá Parthenope, Dip. Scienze Applicate, Centro Direzionale Isola C4, 80143 Napoli, Italy \\ Received 14 May 2010 / Accepted 14 September 2010
}

\begin{abstract}
Aims. The aim of this paper is to contribute to the characterisation of the general properties, differences, and commonalities of the short period comet family and, more in particular, to characterise the dust environment of three poorly studied members of the family: 32P/Comas Solá, 56P/Slaughter-Burnham, and 78P/Gehrels 2.

Methods. Multicolour broad-band photometry of the comets observed around their perihelion in October 2004 from the Loiano telescope has been used to characterise their dust coma morphology and properties and to model the dust production rate

Results. 32P/Comas Solá shows no remarkable dust coma features, while both 56P/Slaughter-Burnham and 78P/Gehrels 2 show departures from regular brightness distribution, with a north/south asymmetry and axysimmetric lobes, respectively, probably indicating emission features on the nucleus surface. For comet 78P, it has been possible to compute a 1-D surface brightness profile with slope $m=-1.54 \pm 0.05$. In an aperture of radius $\rho=7.2 \times 10^{3} \mathrm{~km}$, the measured Af $\rho$ is $127 \pm 10 \mathrm{~cm}, 102 \pm 8 \mathrm{~cm}$ (weighted average between two observing nights) and $846 \pm 55 \mathrm{~cm}$ for comets 32P, 56P and 78P, respectively. All comets have a redder dust coma than the Sun. Comets $32 \mathrm{P}$ (dust production rate $Q_{\mathrm{d}}$ from 2 to $\left.46 \mathrm{~kg} / \mathrm{s}\right)$ and $56 \mathrm{P}\left(Q_{\mathrm{d}}\right.$ from 1 to $\left.24 \mathrm{~kg} / \mathrm{s}\right)$ are quite standard dust emitters among the short-period comet family, while comet $78 \mathrm{P}\left(Q_{\mathrm{d}}\right.$ from 14 to $\left.345 \mathrm{~kg} / \mathrm{s}\right)$ is more active than the average at that heliocentric distance.
\end{abstract}

Key words. comets: general - comets: individual: 32P/Comas Solá - comets: individual: 56P/Slaughter-Burnham comets: individual: $78 \mathrm{P} /$ Gehrels 2

\section{Introduction}

Comets are commonly considered the remnants of the original proto-solar disk from which our Solar System formed. Spending the majority of their lives in the coldest region of the planetary system, cometary nuclei are likely composed of relatively unprocessed material and may even preserve presolar grains. Understanding their physical and compositional properties is crucial for shedding light on formation and evolution processes that could take place when forming a planetary system, e.g., the radial mixing in the solar nebula from the hottest internal regions to the colder outer part beyond Neptune (Brownlee et al. 2006).

Direct imaging and photometry of cometary coma is a powerful tool for investigating the intrinsic coma properties and morphology, so far characterising the cometary dust environment and the differences between different classes of minor bodies. Many comets are known to exhibit distinct features (jets, spikes, arcs, shells, etc.) in their comae, which are generally believed either to result from the ejection of material from discrete active areas on the nucleus or to be the direct consequence of particular topographic structure over the nucleus surface. See the recent review by Farnham (2009) for a discussion of how particular features are observed and interpreted to derive properties relating to the comet. The frequent formation of distinct features in the dust coma and its possible relation with the formation of gas features is not yet well understood. No unique solution has been found to explain coma morphology and its evolution for all comets, so it needs to be studied in many targets, in order to investigate commonalities and differences for different cometary classes (see e.g. Schulz 2002).

Short-period comets (orbital period less than 20 years) are a class of minor bodies of particular interest, since their characteristics can be studied during several passages along the Solar System, and the processes governing the evolution of their dust and gas environment can be directly monitored. It has been realised over the years that the evolution of the activity of a comet as it moves along its orbit is of utmost importance for understanding the structure and composition of its nucleus. Moreover, gathering information on cometary dust production rate and its evolution along the orbital path and over several orbital passages allows constraints to be added to models describing the evolution of different small body families in the Solar System and their contribution to the interplanetary dust complex (Sykes et al. 2004).

32P/Comas Solá, 56P/Slaughter-Burnham and 78P/Gehrels 2 are poorly known short-period comets of the Jupiter family class. 32P/Comas Solá was discovered by J. Comas Solá on 4 November 1926, on photographs taken at the Barcelona Fabra Observatory. The comet is classified as "erratic", as its long-term non-gravitational perturbations present rapidly varying discontinuities (Królikowska et al. 1998). Sekanina (1993) has suggested that the comet behaviour, observed for most of the comet perihelion passages, can be explained by outgassing from a single small active area with a modest precession rate, but evolution of the motion seems also to suggest that active regions on the 
A\&A 525, A62 (2011)

Table 1. Orbital characteristics and elements of the target comets. All data from the JPL Horizon Database.

\begin{tabular}{ccccccccc}
\hline \hline Comet & $P^{a}[\mathrm{y}]$ & $\tau^{b}$ & $q^{c}[\mathrm{AU}]$ & $e^{d}$ & $\omega^{e}\left[{ }^{\circ}\right]$ & $\Omega^{f}\left[{ }^{\circ}\right]$ & $i^{g}\left[^{\circ}\right]$ & $T_{J}{ }^{h}$ \\
\hline 32P/Comas Solá & 8.80 & 1 Apr. 2005 & 1.83 & 0.569 & 45.93 & 60.79 & 12.93 & 2.67 \\
56P/Slaughter-Burnham & 11.54 & 15 Jan. 2005 & 2.54 & 0.504 & 44.08 & 346.27 & 8.16 & 2.71 \\
78P/Gehrels 2 & 7.22 & 27 Oct. 2004 & 2.01 & 0.463 & 192.97 & 210.54 & 6.25 & 2.89 \\
\hline
\end{tabular}

Notes. ${ }^{(a)}$ Orbital period ${ }^{(b)}$ Perihelion time closest to the time of observations . ${ }^{(c)}$ Perihelion distance . ${ }^{(d)}$ Orbital eccentricity . ${ }^{(e)}$ Perihelion argument (J2000). ${ }^{(f)}$ Nodal line argument (J2000) . ${ }^{(g)}$ Orbital plane inclination (J2000) . ${ }^{(h)}$ Tisserand parameter.

Table 2. Comet geometry during the observations.

\begin{tabular}{ccccccc}
\hline \hline Comet & $\begin{array}{c}\text { Obs. night } \\
(2004)\end{array}$ & $r^{a}[\mathrm{AU}]$ & $\Delta^{b}[\mathrm{AU}]$ & $\mathrm{PA}^{c}$ & $\alpha^{d}\left[^{\circ}\right]$ & Filters $^{e}$ \\
\hline 32P/Comas Solá & 6 Oct. & 2.437 & 1.559 & 266.8 & 14.1 & $B, V, R, I$ \\
56P/Slaughter-Burnham & 5 Oct. & 2.649 & 1.686 & 75.9 & 7.3 & $B, V, R$ \\
& 6 Oct. & 2.647 & 1.689 & 75.5 & 7.7 & $R$ \\
78P/Gehrels 2 & 5 Oct. & 2.016 & 1.148 & 256.5 & 18.8 & $B, V, R, I$ \\
\hline
\end{tabular}

Notes. ${ }^{(a)}$ Heliocentric distance . ${ }^{(b)}$ Geocentric distance . ${ }^{(c)}$ Position angle of the extended Sun-target radius vector . ${ }^{(d)}$ Phase angle . ${ }^{(e)}$ Broadband filters used for the observations .

nucleus surface could be short-lived sources of non-gravitational disturbance. Królikowska et al. (2001), by applying the forced precession model of a non-spherical nucleus to all the available updated astrometric data, suggest that the nucleus of 32P/Comas Solá, with the ratio of equatorial to polar ratio of 0.78 , is the most prolate one in the erratic class.

56P/Slaughter-Burnham was serendipitously discovered by C. D. Slaughter and R. Burnham (Lowell Observatory, Arizona) during the course of the Proper Motion Survey on January 1959. Little information is available on this target. It is known to be one of the only three SPCs in a $1 / 1$ resonance with Jupiter (Benest $\&$ Gonczi 1992), and this has stochastic effects on its dynamical orbital parameters.

78P/Gehrels 2 was discovered by Tom Gehrels while examining plates exposed with Palomar Observatory's $122 \mathrm{~cm}$ Schmidt Telescope during a minor planet survey in September and October 1973. The short-period nature of its orbit was then confirmed, with an orbit indicating that the comet had passed very close to Jupiter in 1971, so decreasing its perihelion distance to the actual value.

All the three comets have only been studied (snapshot observations) when at large distance (i.e., >3 AU) from the Sun: comet 32P/Comas Solá was imaged at heliocentric distance $r=$ 3.1 AU (Lowry et al. 1999), and it was found to be active. Comet 56P/Slaughter-Burnham was imaged at $r=3.8$ AU (Snodgrass et al. 2008) and $r=7.4 \mathrm{AU}$ (Meech et al. 2004): it was detected as an active and stellar object, respectively. From the latter observation, a nucleus radius of $R_{\mathrm{N}}=1.56 \mathrm{~km}$ was derived (for a standard albedo value of 0.04 ). Comet $78 \mathrm{P} /$ Gehrels 2 was imaged at $r=3.8 \mathrm{AU}$ (Snodgrass et al. 2008) and at $r=5.5 \mathrm{AU}$ (Lowry \& Weissman 2003) and it was detected as an active and stellar object. From the latter observation, a nucleus radius of $R_{\mathrm{N}}=1.42 \pm 0.12 \mathrm{~km}$ was derived (for a standard albedo value of $0.04)$. To our knowledge, none of the comets was ever observed to perform a quantitative analysis of coma properties (morphology, dust production, colours, etc.): results presented in this paper are the first available for these targets.

The image observations and their data reduction are presented in Sect. 2. Section 3 contains the observational results, while the dust mass loss rates derived from cometary photometry are discussed in Sect. 4. Summary and conclusions are given in Sect. 5.

\section{Observations and data reduction}

The short-period comets 32P/Comas Solá, 56P/SlaughterBurnham, and 78P/Gehrels 2 were observed with the $1.52 \mathrm{~m}$ Mt. Orzale Cassini telescope at the Bologna Astronomical Observatory of Loiano, Italy, on the nights of 5 and 6 October 2004, when the comets were quite close to their perihelion. Orbital characteristics and elements for the target comets are summarised in Table 1. CCD images were obtained using the BFOSC (Bologna Faint Object Spectrograph and Camera) instrument, equipped with the EEV D129915 $1300 \times 1340 \mathrm{CCD}$ (pixel size $0.58^{\prime \prime}$, FOV $13^{\prime} \times 12.6^{\prime}$ ) and the Johnson-Kron-Cousin broadband filters $B, V, R$ and $I$. The circumstances of the observations are summarised in Table 2.

The Loiano Cassini telescope is not equipped with a differential tracking guidance system, which could allow the comet to be followed in its proper motion for a long exposure time. Therefore, several short exposure time images were acquired for each comet in each filter. The single exposure time depended on the apparent velocity of the comet compared with the PFOV of the telescope in combination with the seeing of each night, and ranged from 30 up to $180 \mathrm{~s}$. After bias correction and flat fielding, the position of the comet optocentre in each single image was determined by fitting a two-dimensional Gaussian to the 36 innermost pixels of the coma in the cases of comet $78 \mathrm{P} /$ Gehrels 2 or to the whole coma in the case of faintest comets $56 \mathrm{P} /$ Slaughter-Burnham and 32P/Comas-Solá. Then the images were recentred, and those taken with the same filter on each night were summed. Sky correction was performed by subtracting a first-order polynomial sky approximation computed from the pixel areas of the same comet image, both containing no stars and far away from a possible coma contamination. All reduction processes were done with the aid of ESO's MUNICH IMAGE DATA ANALYSIS SYSTEM, MIDAS (1998). To perform an absolute flux calibration of the comet images, appropriate fields (G93-48, SA 92, PG 2331, PG 0231) from the list of Landolt (1992) were observed at different airmasses each night. The seeing was good during the first night $\left(\sim 1.3^{\prime \prime}\right)$, discrete during the second one $\left(\sim 2.0^{\prime \prime}\right)$, when also some cirrus were present in the second part of the night. Table 3 summarises the final images obtained during the whole observing run, after the reduction 
Table 3. Summary of the images obtained during the whole observing run.

\begin{tabular}{cccccc}
\hline \hline Comet & Filter & Total exposure time [s] & UT starting time of observation & Airmass $^{a}$ & Nr. of images $^{-}$ \\
\hline 32P/Comas Solá & $B$ & 1620 & 7 Oct. 2004 01:46 & 1.234 & 9 \\
& $V$ & 960 & 7 Oct. 2004 02:21 & 1.264 & 8 \\
& $R$ & 1200 & 7 Oct. 2004 00:40 & 1.233 & 12 \\
& $I$ & 720 & 7 Oct. 2004 01:30 & 1.222 & 6 \\
56P/Slaughter-Burnham & $B$ & 1320 & 5 Oct. 2004 22:16 & 1.410 & 11 \\
& $V$ & 660 & 5 Oct. 2004 21:55 & 1.388 & 11 \\
& $R$ & 1020 & 5 Oct. 2004 21:15 & 1.385 & 17 \\
78P/Gehrels 2 & $R$ & 1320 & 6 Oct. 2004 19:16 & 1.638 & 22 \\
& $B$ & 240 & 6 Oct. 2004 03:25 & 1.217 & 4 \\
& $V$ & 240 & 6 Oct. 2004 03:17 & 1.203 & 4 \\
& $R$ & 1080 & 6 Oct. 2004 02:54 & 1.181 & 18 \\
& $I$ & 600 & 6 Oct. 2004 02:33 & 1.151 & 10 \\
\hline
\end{tabular}

Notes. ${ }^{(a)}$ Median value for the total exposure time (coadded image).

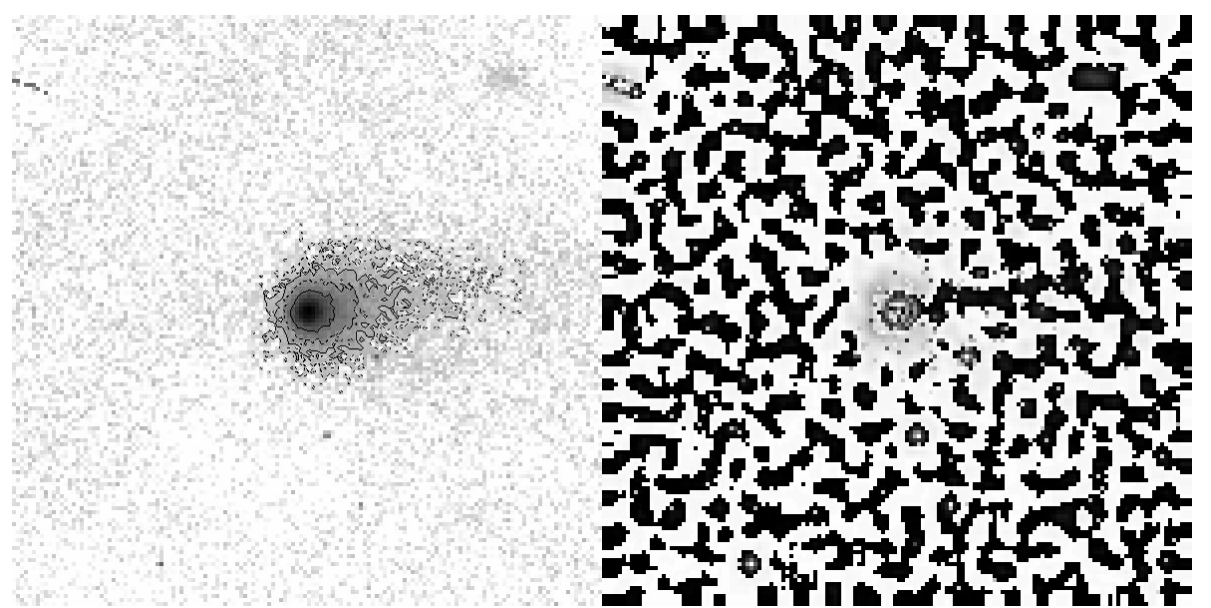

Fig. 1. (left) Final coadded image in the $B$ filter for comet 32P/Comas Solá, obtained on 6 October 2004, with overlayed contour plot. The faintest plotted isophote corresponds to $24.13 B$-mag/ $\operatorname{arcsec}^{2}$. The total scale of the image is $1.4^{\prime}$, which corresponds to a linear scale of $9.7 \times 10^{4} \mathrm{~km}$ at the comet. $\mathrm{N}$ is down, $\mathrm{E}$ to the left. The Sun is $3.2^{\circ} \mathrm{N}$ of $\mathrm{E}$ (PA of the comet: $266.8^{\circ}$ ). (right) Corresponding processed image (Laplace filter with a filter width of 11 pixels).

processes, and used for the analysis described in the following sections.

\section{Observational results}

Images obtained during the whole observing run are presented in Figs. 1 to 6, together with the corresponding processed images. The original (coadded) images are shown on a logarithmic display scale to enhance the faintest brightness levels.

\subsection{Coma photometry and morphology}

The coma integrated magnitudes, derived for all the filters in some selected optical apertures centred in the optocentre (up to a radius of $\sim 10^{\prime \prime}$ ), are listed in Table 4 . For each photometric aperture, the final uncertainty in the magnitude comes from the sum of the photometric error $\sigma_{\text {count }}$ (comet counts against the background in the original image) and the calibration error $\sigma_{\text {calib }}$ in the calibration curve, taken as the rms of the standard stars fit, weighted by the star counts against the background. Figure 7 allows comparison of the measured integrated magnitude at different photometric apertures for all the target comets, in each filter (for each filter, the value has been measured and listed up to an aperture radius where the counts were just above $1 \sigma$ of the background). For further analysis and comparison among the comets, the same physical aperture of radius $7.2 \times 10^{3} \mathrm{~km}$ has been chosen for all the targets. In our opinion this allows a direct comparison of coma photometric properties based on a fixed physical parameter, the (projected) size of the expanding coma.

Coma morphology is a powerful tool to investigate the nucleus and coma properties, by observing and interpreting different features such as e.g. bright jets, fans, and arc structures (see the recent review by Farhnam 2009). We investigated the coma images to search for distinct dust extended features. For this purpose we applied several analysis techniques to the coma images, depending on image quality and signal-to-noise ratio (SNR). The so-called adaptive Laplace filtering method (Boehnhardt \& Birkle 1994) is sensitive to gradient change on different spatial scales depending on the width of the spatial filter applied. It has been successfully applied to cometary broad-band images (e.g., Boehnhardt \& Birkle 1994; Lara et al. 2004a,b) to enhance weak dust (and gas) cometary features. We applied it to all the (coadded) images obtained during the run, with an example shown in Fig. 1. This method did not reveal any feature for the three comets.

The so-called radial normalisation method (Birkle \& Boehnhardt 1992) reduces the general coma background and 


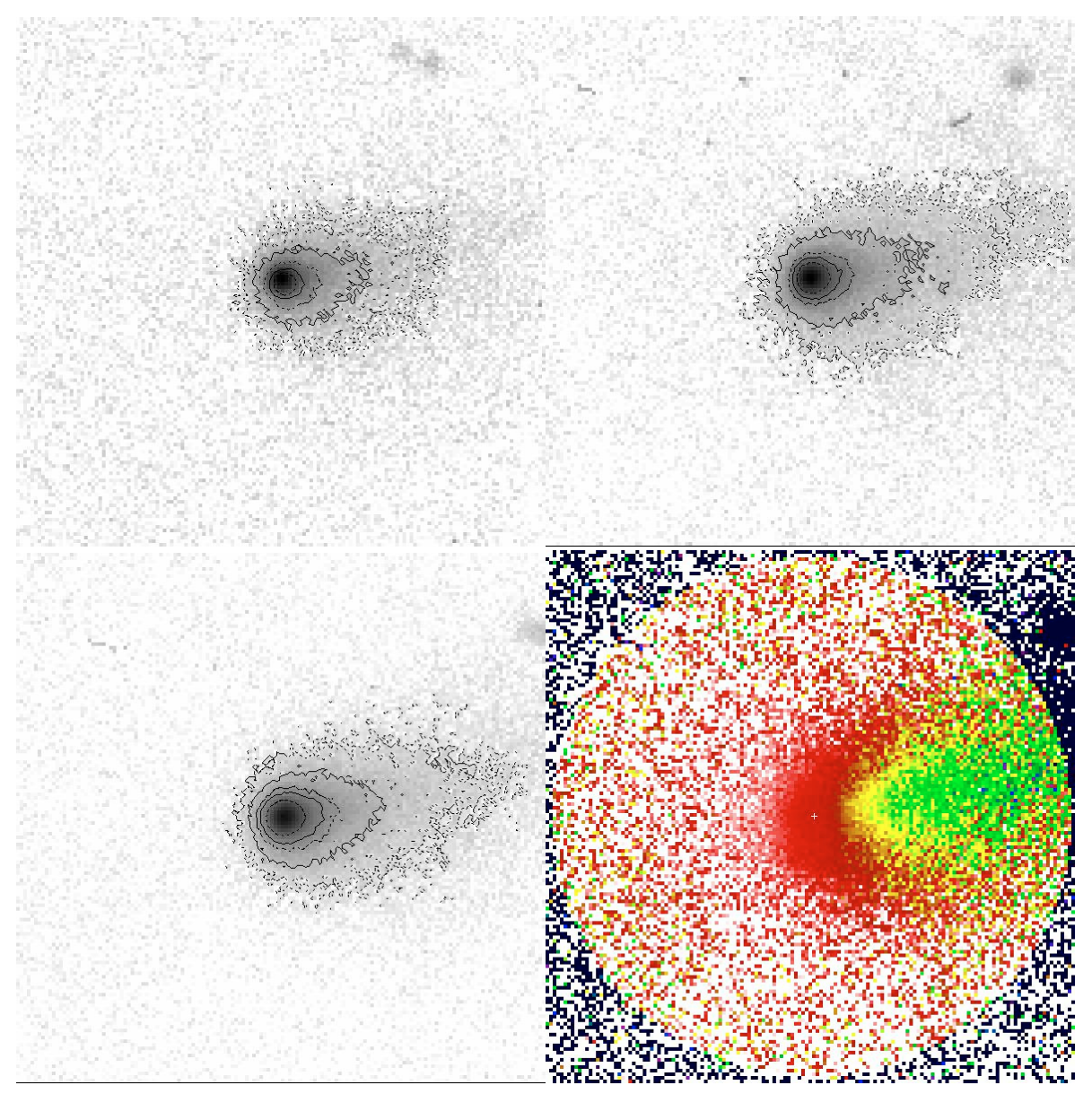

Fig. 2. For all the filters, the total scale of the image is $1.4^{\prime}$, which corresponds to a linear scale of $9.7 \times 10^{4} \mathrm{~km}$ at the comet. $\mathrm{N}$ is down, $\mathrm{E}$ to the left. The Sun is $3.2^{\circ} \mathrm{N}$ of $\mathrm{E}$ (PA of the comet: $266.8^{\circ}$ ). (top left) Final coadded image in the $V$ filter for comet 32P/Comas Solá, obtained on the night of 6 October 2004, with overlayed contour plot. The faintest plotted isophote corresponds to $23.63 \mathrm{~V}$-mag/arcsec ${ }^{2}$. (top right) Final coadded image in the $I$ filter for comet 32P/Comas Solá, obtained on the night of 6 October 2004, with overlayed contour plot. The faintest plotted isophote corresponds to $23.05 \mathrm{I}-\mathrm{mag} / \mathrm{arcsec}^{2}$. (bottom left) Final coadded image in the $R$ filter for comet 32P/Comas Solá, obtained on the night of 6 October 2004, with overlayed contour plot. The faintest plotted isophote corresponds to $23.41 R$-mag/arcsec ${ }^{2}$. (bottom right) The final $R$ image enhanced by means of the radial renormalization method. The white + marks the optocentre.

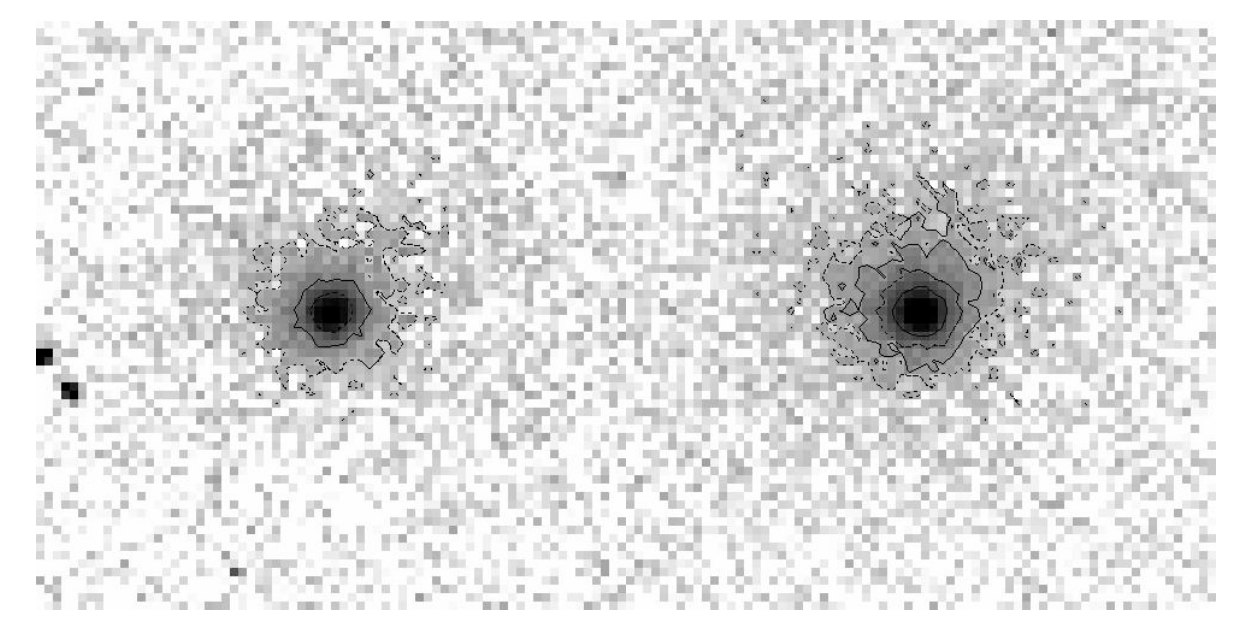

Fig. 3. For both the images, the total scale of the image is $40.6^{\prime \prime}$, which corresponds to a linear scale of $4.9 \times 10^{4} \mathrm{~km}$ at the comet. $\mathrm{N}$ is down, $\mathrm{E}$ to the left. The Sun is $14.1^{\circ} \mathrm{S}$ of W (PA of the comet: $75.9^{\circ}$ ). (left) Final coadded image in the $B$ filter for comet 56P/Slaughter-Burnham, obtained on the night of 5 October 2004, with overlayed contour plot. The faintest plotted isophote corresponds to 23.68 B-mag/arcsec ${ }^{2}$. (right) Final coadded image in the $V$ filter for comet 56P/Slaughter-Burnham, obtained on the night of 5 October 2004, with overlayed contour plot. The faintest plotted isophote corresponds to $22.98 \mathrm{~V}$-mag/ $\operatorname{arcsec}^{2}$.

enhances faint coma structures. It has been repeatedly applied to cometary broad-band images (e.g., Birkle \& Boehnhardt 1992; Fulle et al. 1993; Lara et al. 2009). For the purpose of the present analysis, it was applied with the following procedure: (1) the mean radial brightness in concentric rings of 1 pixel width was computed around the centre of the coma, obtaining an artificial 


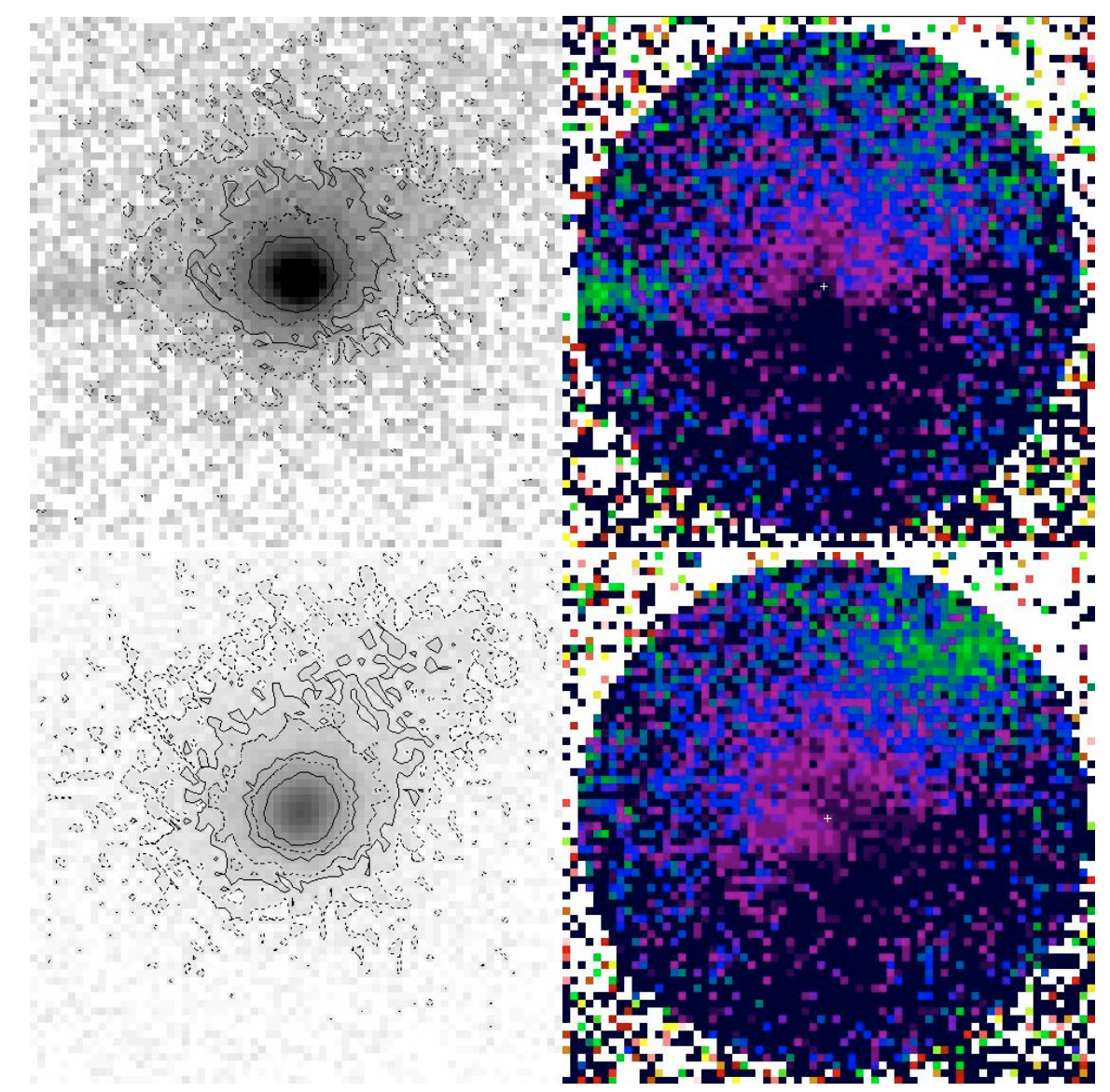

Fig. 4. For all the images, the total scale of the image is $40.6^{\prime \prime}$, which corresponds to a linear scale of $4.9 \times 10^{4} \mathrm{~km}$ at the comet. $\mathrm{N}$ is down, $\mathrm{E}$ to the left. The Sun is $14.1^{\circ} \mathrm{S}$ of W (PA of the comet: $75.9^{\circ}$ ). (top left) Final coadded image in the $R$ filter for comet $56 \mathrm{P} / \mathrm{Slaughter}-\mathrm{Burnham}$, obtained on the night of 5 October 2004, with overlayed contour plot. The faintest plotted isophote corresponds to $23.36 R$-mag/arcsec ${ }^{2}$. (top right) The same image enhanced by means of radial renormalisation method. The white + marks the optocentre. (bottom left) Final coadded image in the $R$ filter for comet 56P/Slaughter-Burnham, obtained on the night of 6 October 2004, with overlayed contour plot. The faintest plotted isophote corresponds to $23.37 R$-mag/arcsec ${ }^{2}$. (bottom right) The same image enhanced by means of radial renormalisation method. The white + marks the optocentre.

Fig. 5. For both the images, the scale of the image is $1.4^{\prime}$, which corresponds to a linear scale of $7.2 \times 10^{4} \mathrm{~km}$ at the comet. $\mathrm{N}$ is down, E to the left. The Sun is $13.5^{\circ} \mathrm{N}$ of E (PA of the comet: $256.5^{\circ}$ ). (left) Final coadded image in the $B$ filter for comet $78 \mathrm{P} /$ Gehrels 2 , obtained on the night of 5 October 2004, with overlayed contour plot. The faintest plotted isophote corresponds to $21.45 B$-mag/arcsec ${ }^{2}$. (right) Final coadded image in the $V$ filter for comet 78P/Gehrels 2, obtained on the night of 5 October 2004, with overlayed contour plot. The faintest plotted isophote corresponds to $21.69 \mathrm{~V}$-mag/arcsec ${ }^{2}$.

image characterised by the radial dependence of the original image; (2) the division of the original image by the mean radial image allows direct determination of coma magnitude variations relative to the mean radial coma brightness.

\subsubsection{P/Comas Solá}

The obtained coadded images for comet 32P/Comas Solá are shown in Figs. 1 and 2, for all the filters. 32P/Comas Solá was observed at $r=2.44 \mathrm{AU}$ on its inbound branch, with magnitude 


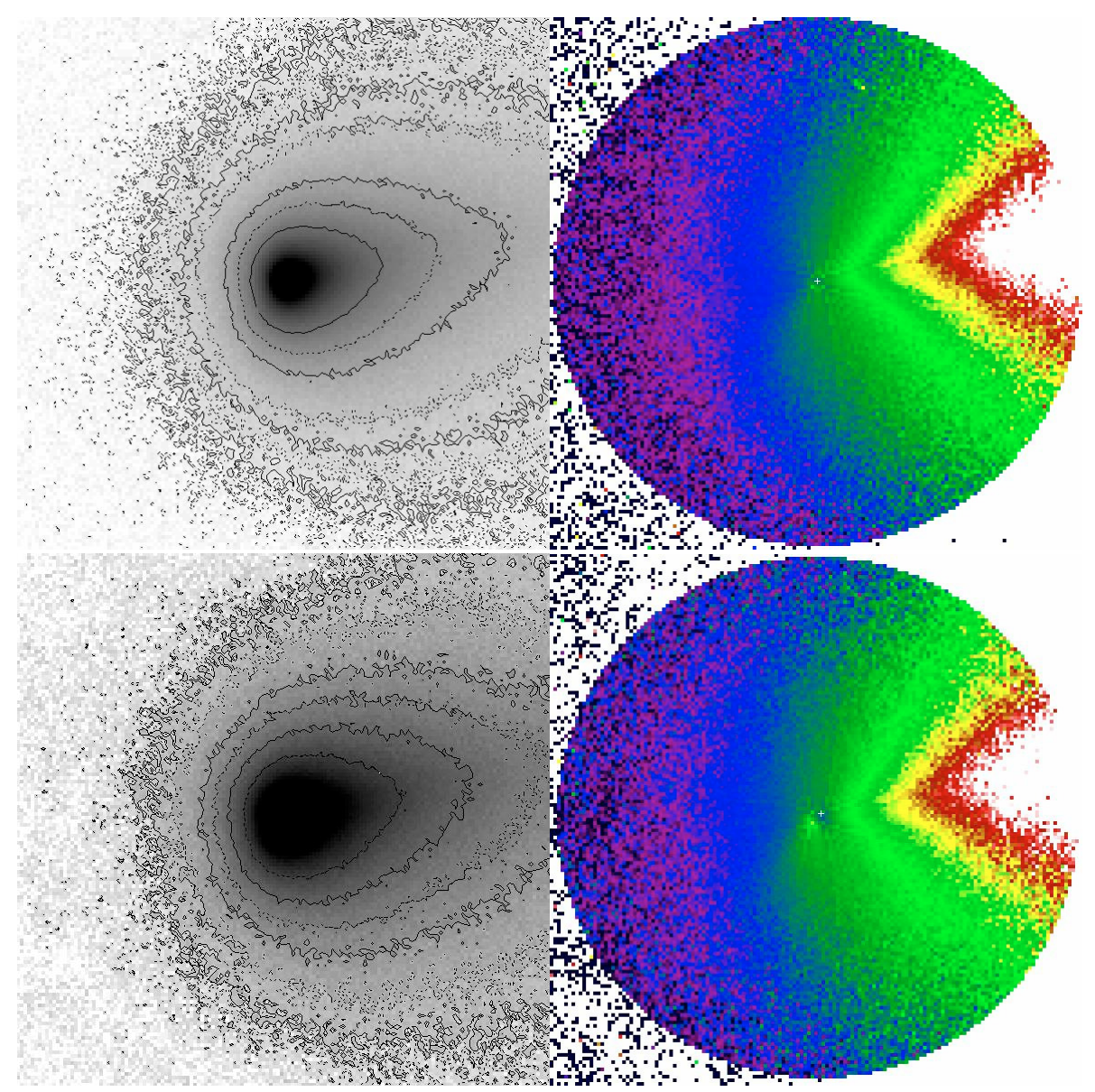

Fig. 6. For all the images, the scale of the image is $1.4^{\prime}$, which corresponds to a linear scale of $7.2 \times 10^{4} \mathrm{~km}$ at the comet. $\mathrm{N}$ is down, E to the left. The Sun is $13.5^{\circ} \mathrm{N}$ of $\mathrm{E}$ (PA of the comet: $256.5^{\circ}$ ). (top left) Final coadded image in the $R$ filter for comet 78P/Gehrels 2, obtained on the night of 5 October 2004, with overlayed contour plot. The faintest plotted isophote corresponds to $23.06 R$-mag/arcsec ${ }^{2}$. (top right) The same image enhanced by means of the radial renormalisation method. The white + marks the optocentre. (bottom left) Final coadded image in the $I$ filter for comet 78P/Gehrels 2, obtained on the night of 5 October 2004, with overlayed contour plot. The faintest plotted isophote corresponds to 22.24 $I$-mag/arcsec ${ }^{2}$. (bottom right) The same image enhanced by means of the radial renormalisation method. The white + marks the optocentre.

$B=17.136 \pm 0.264, V=16.290 \pm 0.137, R=15.778 \pm 0.090$ and $I=15.256 \pm 0.088$ in an aperture of radius $\rho=7.2 \times 10^{3} \mathrm{~km}$.

The comet looks active, with a well-developed coma persistent in all the filters, and shows a broad tail-like structure, extending approximately in the $\mathrm{W}$ direction. This is the only remarkable feature observable in the coma, as evident from the Laplace filtered images and more in detail from the radially normalised image ( $R$ filter, Fig. 2).

\subsubsection{P/Slaughter-Burnham}

The obtained coadded images for comet 56P/SlaughterBurnham are shown in Figs. 3 to 4 , for the filters $B, V, R$ in the first observing night, and only $R$ for the second observing night. 56P/Slaughter-Burnham was observed at $r=2.65 \mathrm{AU}$ on its inbound branch, with magnitude $B=17.674 \pm 0.247$, $V=16.825 \pm 0.168, R=16.314 \pm 0.124$ in the first observing night, and $R=16.437 \pm 0.112$ in the second observing night, in an aperture of radius $\rho=7.1 \times 10^{3} \mathrm{~km}$.

The comet looks active in each single frame, even if quite faint against the background. The resulting coadded images in all filters show a regular coma with almost circular isophotes. No medium or large-scale non-spherical structure was evident from the Laplacian filtered images. To enhance faint subtle features, the so-called radial normalisation method
(Birkle \& Boehnhardt 1992) was applied to the $R$ images (presenting in general the highest quality contrast). The results of the enhancing process shown in Fig. 4 indeed indicate a departure (even quite faint) from a "regular" brightness distribution: it is a north/south asymmetry in the dust distribution, persistent over the two nights. It could be interpreted as a dust jet (possibly coming from an active area on the nucleus) pointing approximately in the sun direction and then curved back, but unfortunately the quality of the images is not sufficient to quantitatively analyse of the feature and to derive information on the cometary rotation state. The comet is presently at its aphelion, therefore it could be an interesting target during its next perihelion passage (in 2015).

\subsection{3. $78 \mathrm{P} /$ Gehrels 2}

The obtained coadded images for comet 78P/Gehrels 2 are shown in Figs. 5 and 6. 78P/Gehrels was observed only few days after its perihelion, when it was also at the very close geocentric distance of 1.1 AU. It showed therefore as a bright object, with magnitude $B=13.952 \pm 0.118, V=13.209 \pm 0.095$, $R=12.730 \pm 0.071$, and $I=12.341 \pm 0.081$ in an aperture of radius $\rho=7.2 \times 10^{3} \mathrm{~km}$.

The comet shows a well-developed coma and a broad taillike structure, extending approximately to $\mathrm{W}$, compatible with the antisolar direction (PA of the Comet $256.5^{\circ}$ ). The images 
E. Mazzotta Epifani and P. Palumbo: Dust environment of SPCs 32P/C S, 56P/S-B and 78P/G 2

Table 4. Integrated coma magnitude for the target comets, derived for all the filters in selected optical apertures centred on the optocentre.

\begin{tabular}{|c|c|c|c|c|c|c|}
\hline \multirow[t]{2}{*}{ Comet } & \multirow[t]{2}{*}{$\phi^{a}\left[{ }^{\prime \prime}\right]$} & \multirow{2}{*}{$\begin{array}{c}\rho \\
{\left[10^{3} \mathrm{~km}\right]}\end{array}$} & \multicolumn{4}{|c|}{ Integrated magnitude in different filters } \\
\hline & & & $B$ & V & $R$ & $I$ \\
\hline \multirow[t]{6}{*}{ 32P/Comas Solá } & 1.16 & 1.3 & $19.44 \pm 0.45$ & $18.41 \pm 0.24$ & $18.15 \pm 0.18$ & $17.61 \pm 0.17$ \\
\hline & 3.48 & 3.9 & $17.72 \pm 0.30$ & $16.86 \pm 0.16$ & $16.40 \pm 0.11$ & $15.87 \pm 0.10$ \\
\hline & 5.80 & 6.6 & $17.21 \pm 0.27$ & $16.37 \pm 0.14$ & $15.86 \pm 0.09$ & $15.34 \pm 0.09$ \\
\hline & 6.38 & 7.2 & $17.14 \pm 0.26$ & $16.29 \pm 0.14$ & $15.78 \pm 0.09$ & $15.26 \pm 0.09$ \\
\hline & 8.70 & 9.8 & $\ldots$ & $\ldots$ & $\ldots$ & $\ldots$ \\
\hline & 10.44 & 11.7 & $\ldots$ & $\ldots$ & $\ldots$ & $\ldots$ \\
\hline \multirow{6}{*}{ 56P/Slaughter-Burnham ${ }^{b}$} & 1.16 & 1.4 & $19.25 \pm 0.41$ & $18.54 \pm 0.27$ & $17.99 \pm 0.20$ & $\ldots$ \\
\hline & 3.48 & 4.3 & $18.06 \pm 0.28$ & $17.25 \pm 0.19$ & $16.74 \pm 0.14$ & $\ldots$ \\
\hline & 5.80 & 7.1 & $17.67 \pm 0.25$ & $16.83 \pm 0.17$ & $16.31 \pm 0.12$ & $\ldots$ \\
\hline & 6.38 & 7.8 & $17.61 \pm 0.24$ & $16.75 \pm 0.17$ & $16.24 \pm 0.12$ & $\ldots$ \\
\hline & 8.70 & 10.7 & $\ldots$ & $16.52 \pm 0.16$ & $16.01 \pm 0.12$ & $\ldots$ \\
\hline & 10.44 & 12.6 & $\ldots$ & $\ldots$ & $15.87 \pm 0.11$ & $\ldots$ \\
\hline \multirow[t]{6}{*}{$56 \mathrm{P} /$ Slaughter-Burnham ${ }^{c}$} & 1.16 & 1.4 & $\ldots$ & $\ldots$ & $18.55 \pm 0.22$ & $\ldots$ \\
\hline & 3.48 & 4.3 & $\ldots$ & $\ldots$ & $16.93 \pm 0.13$ & $\ldots$ \\
\hline & 5.80 & 7.1 & $\ldots$ & $\ldots$ & $16.44 \pm 0.11$ & $\ldots$ \\
\hline & 6.38 & 7.8 & $\ldots$ & $\ldots$ & $16.36 \pm 0.11$ & $\ldots$ \\
\hline & 8.70 & 10.7 & $\ldots$ & $\ldots$ & $16.12 \pm 0.10$ & $\ldots$ \\
\hline & 10.44 & 12.6 & $\ldots$ & $\ldots$ & $15.99 \pm 0.10$ & $\ldots$ \\
\hline \multirow[t]{6}{*}{ 78P/Gehrels 2} & 1.16 & 1.0 & $16.22 \pm 0.17$ & $15.45 \pm 0.12$ & $15.06 \pm 0.09$ & $15.54 \pm 0.10$ \\
\hline & 3.48 & 2.9 & $14.80 \pm 0.13$ & $14.06 \pm 0.10$ & $13.59 \pm 0.08$ & $13.20 \pm 0.09$ \\
\hline & 5.80 & 4.8 & $14.31 \pm 0.12$ & $13.57 \pm 0.10$ & $13.09 \pm 0.07$ & $12.70 \pm 0.08$ \\
\hline & 6.38 & 5.2 & $14.22 \pm 0.12$ & $13.49 \pm 0.10$ & $13.00 \pm 0.07$ & $12.62 \pm 0.08$ \\
\hline & 8.70 & 7.2 & $13.95 \pm 0.12$ & $13.21 \pm 0.10$ & $12.73 \pm 0.07$ & $12.34 \pm 0.08$ \\
\hline & 10.44 & 8.7 & $13.80 \pm 0.12$ & $13.05 \pm 0.09$ & $12.58 \pm 0.07$ & $12.19 \pm 0.08$ \\
\hline
\end{tabular}

Notes. ${ }^{(a)}$ Radius of the photometric aperture. ${ }^{(b)}$ Night of 5 Oct. ${ }^{(c)}$ Night of 6 Oct.

(especially those in the $R$ and $I$ filters) show well-defined, parabolic envelopes, centred on the sunward direction. The Laplace filtering applied to the images did not enhance any particular dust feature in the coma, but the more subtle enhancement method of the radial normalisation technique applied to the $R$ and $I$ image (those with the best signal-to-noise ratio) revealed (Fig. 6) the presence of lobes centred on the comet-Sun line. These lobes appear nearly axisymmetric, but for an emission excess in the NE quadrant close to the optocentre, which is more evident at longer wavelength (filter $I$ ). This excess is not present in the single images used for the coadding, so we tend to interpret it as an artifact of the image processing. The lobes look very similar to those observed in comet C/1999 S4 (LINEAR) (Farhnam 2009): in that case, the structures were interpreted as rotationally generated in the presence of a jet, and associated with fragmentation process and/or nucleus splitting, even if a fragment is not detected. In the case of comet 78P/Gehrels 2, such an interpretation is not supported by other independent data or observations, therefore it could be considered in our opinion a hint to further explore the nucleus and the dust environment of this comet during future orbital passages. (The comet is presently on its inward orbital branch and will reach its next perihelion in January 2012).

\subsection{Coma profiles}

For the analysis of the overall aspect of the coma, 1-D surface brightness profiles also can be obtained from the images to test the hypothesis of the "fountain model", i.e., the canonical isotropic steady-state coma (see e.g. Gehrz \& Ney 1992), which should produce a profile with a slope of -1 . When the effect of solar radiation pressure becomes important, the profiles can be as steep as -1.5 (Jewitt and Meech 1987). Steeper slopes may be caused by non-steady state emission, or by fading grains, whereas shallower profiles require a source function in the coma (e.g., fragmenting grains or production of gas from grains).

During the present observing run, only for comet $78 \mathrm{P} /$ Gehrels 2 we obtained $R$-images with SNR high enough to compute the 1-D surface brightness profile, shown in Fig. 8. A linear least-square fit to the data beyond $1.3^{\prime \prime}$ (i.e., outside the area affected by seeing) gives a slope of $m=-1.54 \pm 0.05$, which is just above the limit of a steady-state coma with dominant distortion effect by solar radiation pressure. This slope is consistent with a steady-state coma for comet $78 \mathrm{P} /$ Gehrels 2 . When considering also the morphology analysis described in the previous section, we can say that our data on this comet do not help to distinguish between a steady-state or non-steady-state emission.

\section{3. $A f \rho$}

The Af $\rho$ value [cm] (A'Hearn et al. 1984), where $A$ is the average grain albedo, $f$ the filling factor in the aperture field of view, and $\rho$ the linear radius of the aperture at the comet, i.e., the skyplane radius, is usually used as a proxy for the cometary dust production. When the cometary coma is in a steady-state, A $f \rho$ is an aperture-independent parameter. It is formulated to take the solar flux scattered by the cometary dust towards the observer into account, and can be derived from the calculated photometric dust coma magnitude as follows:

$A f \rho=\frac{4 r^{2} \Delta^{2} 10^{0.4\left(m_{\mathrm{S}}-m_{\mathrm{C}}\right)}}{\rho}$

where $r$ is the heliocentric distance, $\Delta$ the geocentric distance, $m_{\mathrm{S}}$ the Sun magnitude, and $m_{\mathrm{C}}$ the comet magnitude calculated in the aperture of radius $\rho$.

A monotone decrease in A $f \rho$ values with the nucleocentric distance $\rho$ may indicate non-steady-state dust emission and possibly dust grain fading or destruction, as reported for several 

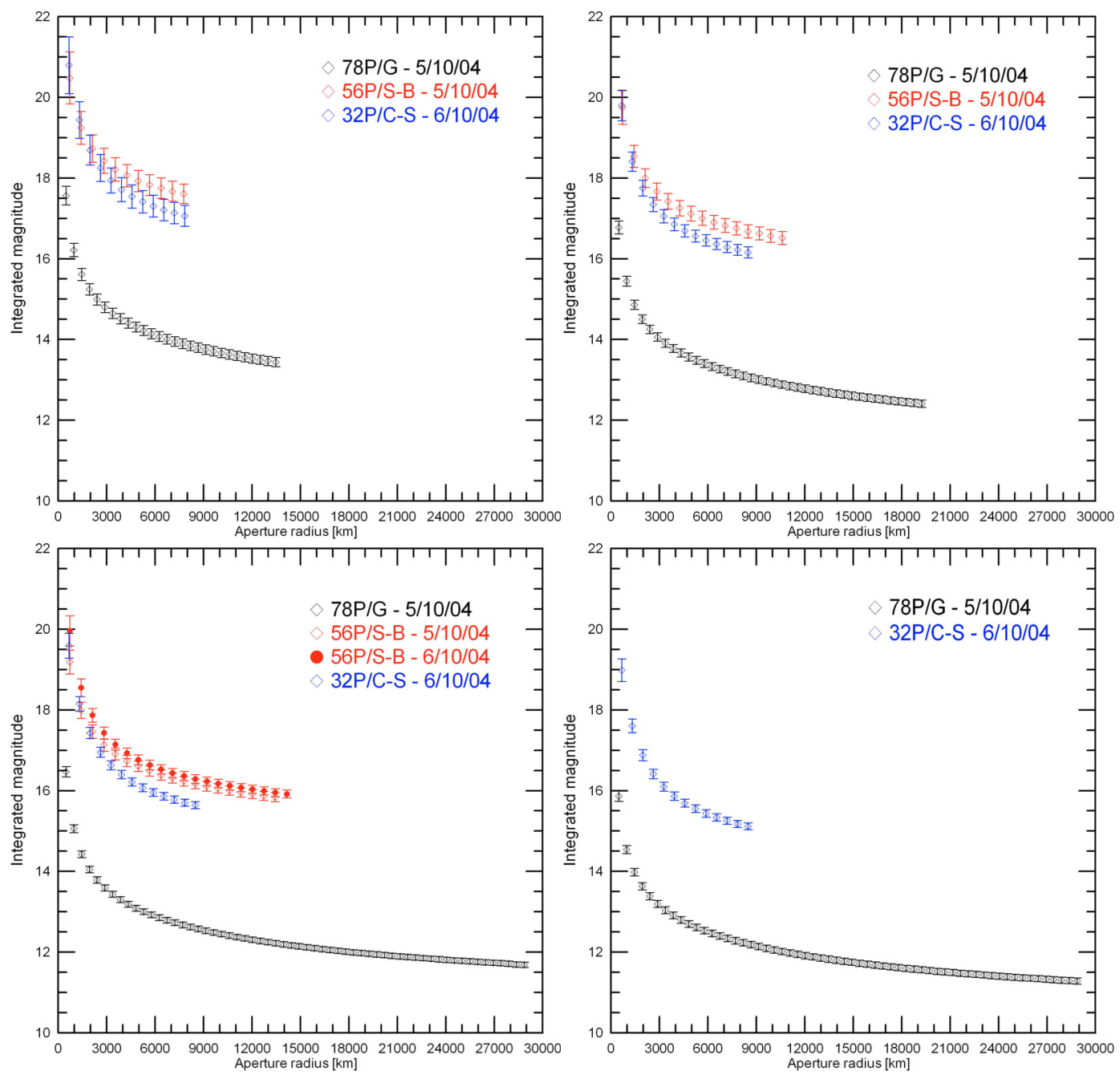

Fig. 7. Comparison of the measured integrated magnitude at different photometric apertures for all the target comets, in different filters: (top left) $B$ filter; (top right) $V$ filter; (bottom left) $R$ filter; (bottom right) $I$ filter.

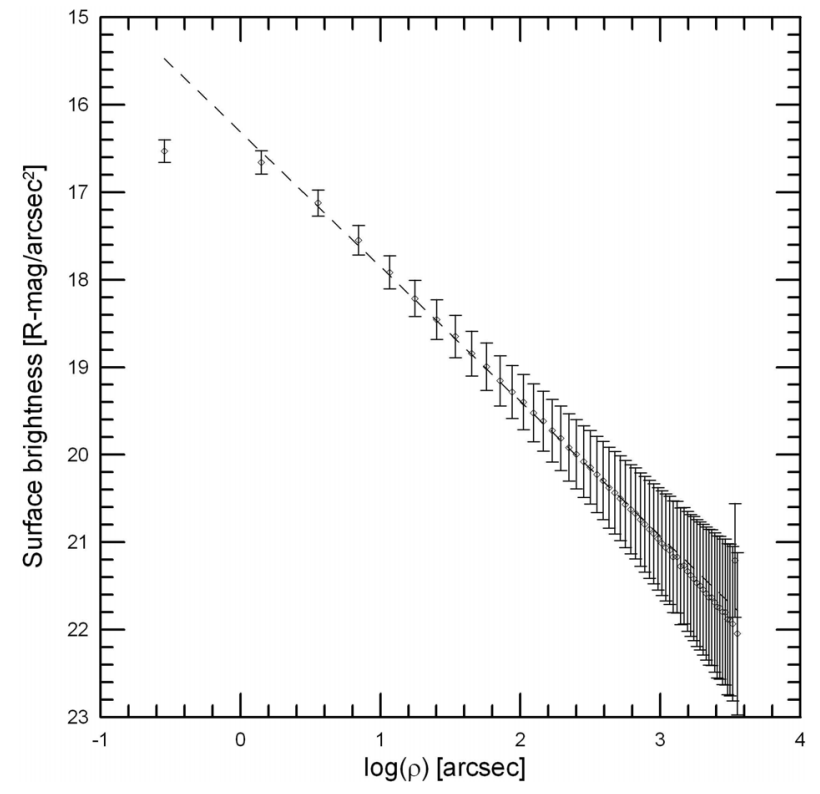

Fig. 8. Surface Brightness Profile (SBP) for comet 78P/Gehrels 2, obtained in the $R$ filter on 5 October 2004. The dashed line is a linear least-square fit to the data beyond 1.3" (i.e., outside the area affected by the seeing), with slope $m=-1.54 \pm 0.05$. comets, such as C/2000 WM1 (LINEAR) (Lara et al. 2004b) and C/2001 Q4 (NEAT) (Tozzi et al. 2003). In contrast, A $\rho$ should be constant at all apertures for an ideal steady-state coma.

The values of A $f \rho$ for the target comets, derived for all the filters in some selected optical apertures centred on the optocentre, are listed in Table 5. The used Loiano $R$ band-pass filter (roughly centred at $\lambda \pm 6000 \AA$, with an FWHM of $\sim 200 \AA$ ) is the least affected by possibly present gaseous emission (next strongest emission bands are those of $\mathrm{CN}[\lambda \sim 3870 \AA], \mathrm{C}_{3}$ $[\lambda \sim 4070 \AA], \mathrm{C}_{2}[\lambda \sim 5100 \AA]$ and $\left.\mathrm{NH}_{2}[\lambda \sim 5700 \AA]\right)$, therefore is the most indicated for a comparison of the dust continuum for the target comets. Figure 9 shows the comparison of the $R$-filter A $f \rho$ values versus the optocentric distance for the three targets. Note the different vertical and horizontal scales, which reflect the differences in the magnitude and coma apparent extension, respectively. Even if with a different quality degree for the three targets, the data show that for all the comets the A $f \rho$ parameter is not constant vs. the cometocentric distance, indicating that none of the observed comae is apparently characterised by a steady-state dust emission. This is more evident for comet $78 \mathrm{P} /$ Gehrels 2 , for which the A $f \rho$ value almost halves on a length scale of $\sim 2 \times 10^{4} \mathrm{~km}$.

The Afo parameter is often used to compare dust production rate between comets, but one should keep in mind that it does 

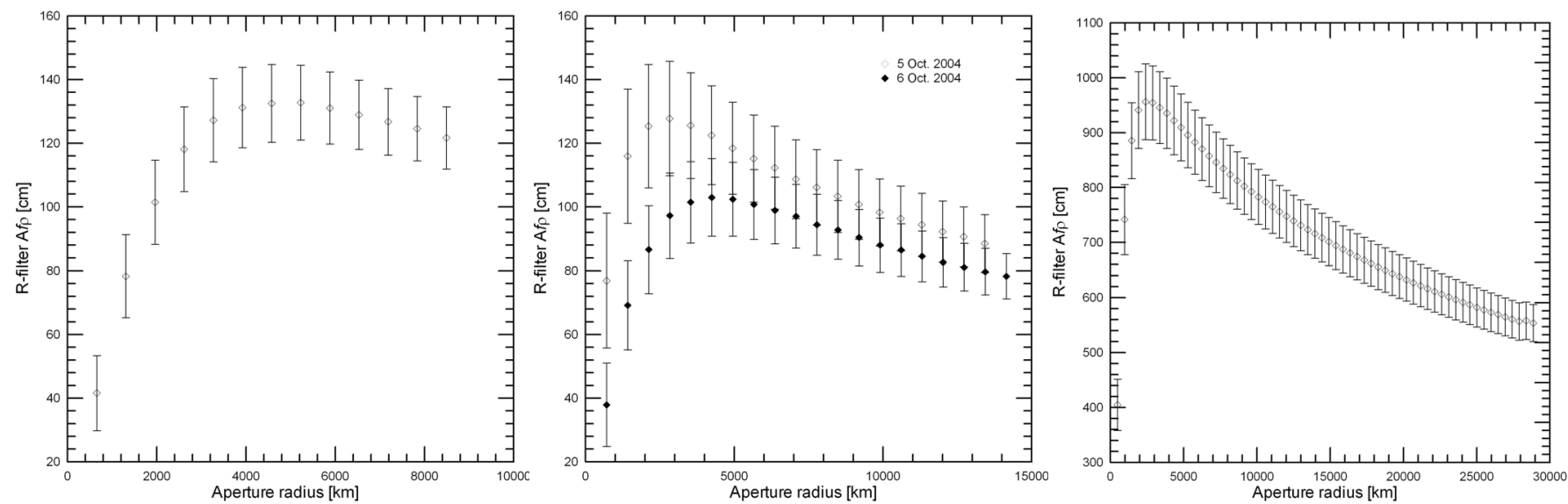

Fig. 9. Comparison of the measured A $f \rho$ value for the target comets versus the optocentre distance. (left) comet 32P/Comas Solá; (middle) comet 56P/Slaughter-Burnham; (right) comet 78PGehrels 2.

Table 5. A $f \rho$ values for the target comets, derived for all the filters in selected optical apertures centred on the optocentre.

\begin{tabular}{|c|c|c|c|c|c|c|}
\hline \multirow[t]{2}{*}{ Comet } & \multirow[t]{2}{*}{$\phi^{a}\left[{ }^{\prime \prime}\right]$} & \multirow{2}{*}{$\begin{array}{c}\rho \\
{\left[10^{3} \mathrm{~km}\right]}\end{array}$} & \multicolumn{4}{|c|}{ A $f \rho$ in different filters $[\mathrm{cm}]$} \\
\hline & & & $B$ & $V$ & $R$ & $I$ \\
\hline \multirow[t]{6}{*}{ 32P/Comas Solá } & 1.16 & 1.3 & $60 \pm 25$ & $86 \pm 19$ & $78 \pm 13$ & $95 \pm 15$ \\
\hline & 3.48 & 3.9 & $98 \pm 27$ & $119 \pm 17$ & $131 \pm 13$ & $157 \pm 15$ \\
\hline & 5.80 & 6.6 & $93 \pm 23$ & $112 \pm 14$ & $129 \pm 11$ & $154 \pm 13$ \\
\hline & 6.38 & 7.2 & $91 \pm 22$ & $109 \pm 14$ & $127 \pm 10$ & $151 \pm 12$ \\
\hline & 8.70 & 9.8 & $\ldots$ & $\ldots$ & $\ldots$ & $\ldots$ \\
\hline & 10.44 & 11.7 & $\ldots$ & $\ldots$ & $\ldots$ & $\ldots$ \\
\hline \multirow[t]{6}{*}{ 56P/Slaughter-Burnham ${ }^{b}$} & 1.16 & 1.4 & $91 \pm 34$ & $97 \pm 24$ & $116 \pm 21$ & $\ldots$ \\
\hline & 3.48 & 4.3 & $91 \pm 23$ & $106 \pm 18$ & $123 \pm 15$ & $\ldots$ \\
\hline & 5.80 & 7.1 & $78 \pm 18$ & $94 \pm 15$ & $109 \pm 12$ & $\ldots$ \\
\hline & 6.38 & 7.8 & $75 \pm 17$ & $91 \pm 14$ & $106 \pm 12$ & $\ldots$ \\
\hline & 8.70 & 10.7 & $\ldots$ & $83 \pm 12$ & $96 \pm 10$ & $\ldots$ \\
\hline & 10.44 & 12.6 & $\ldots$ & $\ldots$ & $91 \pm 9$ & $\ldots$ \\
\hline \multirow{6}{*}{$56 \mathrm{P} /$ Slaughter-Burnham ${ }^{c}$} & 1.16 & 1.4 & $\ldots$ & $\ldots$ & $69 \pm 14$ & $\ldots$ \\
\hline & 3.48 & 4.3 & $\ldots$ & $\ldots$ & $103 \pm 12$ & $\ldots$ \\
\hline & 5.80 & 7.1 & $\ldots$ & $\ldots$ & $97 \pm 10$ & $\ldots$ \\
\hline & 6.38 & 7.8 & $\ldots$ & $\ldots$ & $94 \pm 10$ & $\ldots$ \\
\hline & 8.70 & 10.7 & $\ldots$ & $\ldots$ & $86 \pm 8$ & $\ldots$ \\
\hline & 10.44 & 12.6 & $\ldots$ & $\ldots$ & $81 \pm 7$ & $\ldots$ \\
\hline \multirow[t]{6}{*}{ 78P/Gehrels 2} & 1.16 & 1.0 & $638 \pm 98$ & $719 \pm 82$ & $742 \pm 64$ & $878 \pm 81$ \\
\hline & 3.48 & 2.9 & $786 \pm 95$ & $857 \pm 81$ & $954 \pm 67$ & $1012 \pm 80$ \\
\hline & 5.80 & 4.8 & $741 \pm 84$ & $811 \pm 73$ & $910 \pm 61$ & $958 \pm 73$ \\
\hline & 6.38 & 5.2 & $729 \pm 82$ & $798 \pm 71$ & $896 \pm 60$ & $943 \pm 71$ \\
\hline & 8.70 & 7.2 & $687 \pm 75$ & $754 \pm 66$ & $846 \pm 55$ & $890 \pm 66$ \\
\hline & 10.44 & 8.7 & $660 \pm 71$ & $726 \pm 63$ & $813 \pm 52$ & $856 \pm 63$ \\
\hline
\end{tabular}

Notes. ${ }^{(a)}$ Radius of the photometric aperture. ${ }^{(b)}$ Night of 5 Oct. ${ }^{(c)}$ Night of 6 Oct.

not directly provide a measurement of dust loss. It involves several observational and physical parameters, such as the aperture size (if the A $f \rho$ is not constant with the cometocentric distance, as in the case of the present paper), the geometric albedo of the grains, which depends in turn on the observing phase angle and wavelength. Nevertheless, we selected the aperture with radius $\rho=7.2 \times 10^{3} \mathrm{~km}$ (to minimise effects of the sky background) for all the targets of this paper, to compare their activity using the $R$-band A $\rho$ parameter as a proxy. For comet 32P/Comas Solá, it was $127 \pm 10 \mathrm{~cm}$; for comet $56 \mathrm{P} /$ Slaughter-Burnham, it was $109 \pm 12 \mathrm{~cm}$ on 5 October and $97 \pm 10 \mathrm{~cm}$ on 6 October (weighted average between the two observing nights: $102 \pm 8 \mathrm{~cm}$ ); for comet $78 \mathrm{P} /$ Gehrels $2,846 \pm 55 \mathrm{~cm}$. These values seem consistent with the general trend of increasing A $f \rho$ with decreasing heliocentric distance $r_{\mathrm{h}}$, and, keeping in mind all the caveats listed above, can also be regarded in the framework of the whole family of short period comets. Figure 10 shows the measured all $R$-band A $f \rho$ we found in literature for some short period comets (including one value for comet 32P/Comas Solá at $r_{\mathrm{h}}=3.09 \mathrm{AU}$, Lowry et al. 1999), together with the values obtained in the present paper (and listed above) for our target comets. For comet 32P/Comas Solá, the variation in A $f \rho$ vs. $r_{\mathrm{h}}$, even if referring to two different perihelion passages, is very coherent. For comets 56P/SlaughterBurnham and 78P/Gehrels 2, the values presented in this paper are the first ones available (to our knowledge), and the dust production rate of these comets should be investigated again at different heliocentric distances. This is particularly true for comet $78 \mathrm{P} /$ Gehrels 2, which, according to its A $f \rho$ value at its perihelion, appears to be a very dusty comet (see also below, Sect. 4).

\subsection{Coma colours}

The data obtained during the observation run allowed us to analyse the coma colours of the target comets. Table 6 summarises 
Table 6. Colour indexes for target comets, integrated in the innermost $1.4 \times 10^{4} \mathrm{~km}$ to minimize effects of the sky background.

\begin{tabular}{cccc}
\hline \hline Comet & Colour Index $(B-V)$ & Colour Index $(V-R)$ & Colour Index $(R-I)$ \\
\hline 32P/Comas Solá & $0.85 \pm 0.40$ & $0.51 \pm 0.23$ & $0.52 \pm 0.18$ \\
56P/Slaughter-Burnham & $0.85 \pm 0.42$ & $0.51 \pm 0.29$ & $\ldots$ \\
78P/Gehrels 2 & $0.74 \pm 0.21$ & $0.48 \pm 0.17$ & $0.39 \pm 0.15$ \\
\hline SPC nuclei & & & \\
Solar & $0.82 \pm 0.01$ & $0.47 \pm 0.01$ & $0.46 \pm 0.1$ \\
\hline & 0.642 & 0.354 & 0.332 \\
\hline
\end{tabular}

Notes. The solar colours are those in the modern Johnson-Cousins system (Holmberg et al. 2006). ${ }^{(a)}$ Weighted average of values from the literature, from the compiled list of Lamy et al. 2004 (see their Table 5).

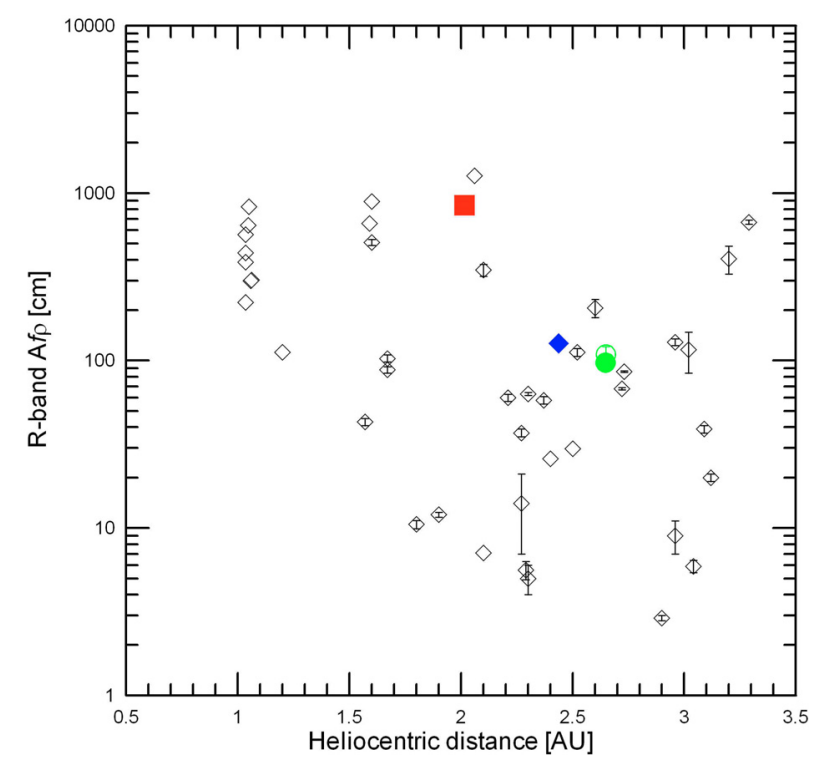

Fig. 10. Measured $R$-band $\mathrm{A} f \rho$ for short period comets. Open black diamonds are values found in literature for comets 1P/Halley (Fink 1994), 4P/Faye (Lamy et al. 1996, 2009), 10P/Tempel 2 (Lamy et al. 2009), 17P/Holmes (Lamy et al. 2009), 21P/Giacobini-Zinner (Lara et al. 2003), 32P/Comas Solá (Lowry et al. 1999), 37P/Forbes (Lamy et al. 2009), 44P/Reinmuth (Lamy et al. 2009), 46P/Wirtanen (Fink et al. 1997, Cartwright et al. 1997, Epifani et al. 1999), 50P/Arend (Lowry and Weissman 2003, Lamy et al. 2009), 59P/Kearns-Kwee (Lamy et al. 2009), 63P/Wild 1 (Lamy et al. 2009), 67P/ChuryumovGerasimenko (Schulz et al. 2004, Lara et al. 2005), 71P/Clark (Lamy et al. 2009), 81P/Wild 2 (Schulz et al. 2003), 84P/Giclas (Lamy et al. 2009), 89P/Russell 2 (Lowry et al. 1999), 106P/Schuster (Lamy et al. 2009), 112P/Urata-Niijima (Lamy et al. 2009), 114P/Wiseman-Skiff (Lamy et al. 2009), 117P/Helin-Roman-Alu 1 (Mazzotta Epifani et al. 2008), 121P/Shoemaker-Holt 2 (Mazzotta Epifani et al. 2008), 137P/Shoemaker-Levy 2 (Lowry et al. 2003). Larger symbols are the values derived in present paper in the innermost $1.4 \times 10^{4} \mathrm{~km}$ : $32 \mathrm{P} /$ Comas Solá at $r_{\mathrm{h}}=2.44 \mathrm{AU}$ (blue filled diamond), 56P/SlaughterBurnham on 5 October 2004 (green open circle) and on 6 October 2004 (green filled circle), 78P/Gehrels 2 (red filled square). The error bars are of the same order or even smaller than the symbol size.

the colours obtained at the optical aperture of radius $\rho=7.2 \times$ $10^{3} \mathrm{~km}$ (i.e., in the innermost $1.4 \times 10^{4} \mathrm{~km}$ ), chosen to minimize the effects of the sky background. This aperture is also suitable for taking most of the coma flux for the faintest comet (56P/Slaughter-Burnham) into account in the faintest filter ( $B$ filter), to allow a straightforward analysis of coma colours ${ }^{1}$. The

\footnotetext{
1 This translates into a different radius of photometric aperture for the three comets: $\rho=6.38^{\prime \prime}$ for comet 32P/Comas Solá, $\rho=$ $5.80^{\prime \prime}$ for comet $56 \mathrm{P} /$ Slaughter-Burnham, and $\rho=8.70^{\prime \prime}$ for comet 78P/Gehrels 2.
}

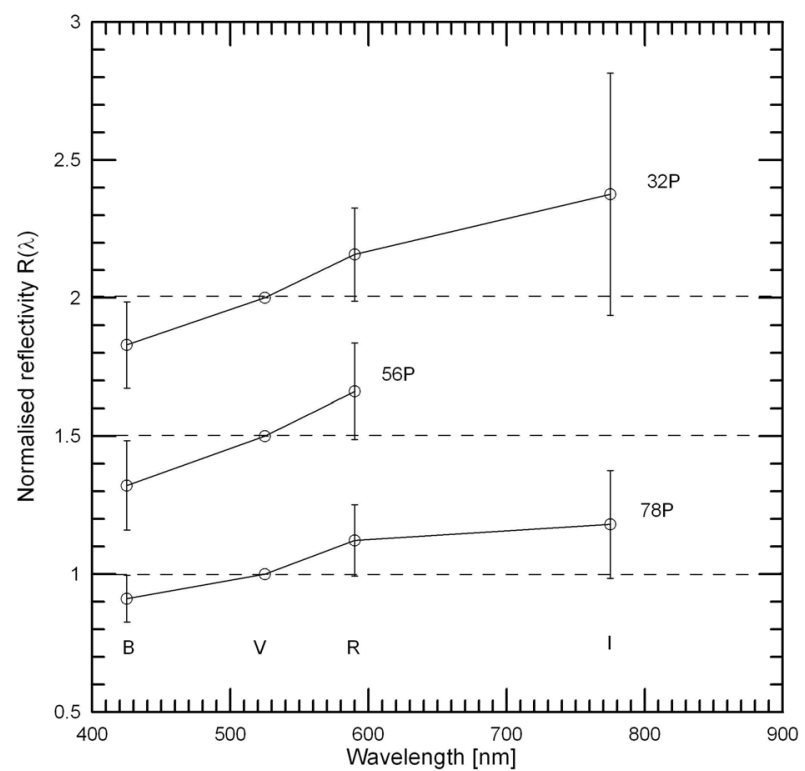

Fig. 11. Normalised reflectivity spectra of target comets. The spectra have been normalised to 1 at $\lambda=525 \mathrm{~nm}$ (band centre of $V$ filter) and vertically shifted for clarity (additive offset). The spectra derive from coma photometry at $\rho=7.2 \times 10^{3} \mathrm{~km}$. The horizontal lines represent a perfectly solar dust.

uncertainties on the colour indexes listed in Table 6 are quite large, especially for the faintest comet 56P/Slauhter-Burnham, nevertheless from the comparison of cometary values with the solar ones we observe that all the three comae are slightly redder than the Sun. Data from Table 6 allow also a tentative comparison of coma colours for our target comets with the average of nucleus colours for the short period comets family (available from the list of Lamy et al. 2004). This comparison should be carefully regarded, since colour index strictly depends on observational and data reduction constraints (e.g., aperture size used to compute the magnitude). Moreover, coma colours are integrated properties, and as such depend significantly on short-term-scale variations in the spatial distribution of dust.

The information contained in the colour indexes can be also converted to a very low-resolution reflectivity spectrum of the cometary coma (Jewitt \& Meech 1986) using the following expression:

$\mathfrak{R}(\lambda)=10^{-0.4\left(m(\lambda)-m_{\mathrm{S}}(\lambda)\right)}$

where $m$ and $m_{\mathrm{S}}$ are the magnitude of the comet and of the Sun ${ }^{2}$. By normalising the reflectivity to 1 at the $V$ central wavelength, it becomes:

$\mathfrak{R}(\lambda)=10^{-0.4((m(\lambda)-m(V))-(m(\lambda)-m(V)) \mathrm{s})}$.

${ }^{2} B_{\odot}=-26.098 ; V_{\odot}=-26.74 ; R_{\odot}=-27.094 ; I_{\odot}=-27.428 ;$ from Holmberg et al. 2006 
Table 7. Spectral reflectivity gradient (reddening) of cometary comae and nuclei.

\begin{tabular}{cccc}
\hline \hline COMETARY COMAE & & & \\
\hline Comet & Spectral range [nm] & Reddening $^{a}[\% / \mathrm{k} \AA$ & Reference \\
\hline 32P/Comas Solá & $525-590$ & $2.54 \pm 0.57$ & this paper \\
& $525-775$ & $1.54 \pm 0.49$ & this paper \\
56P/Slaughter-Burnham & $525-590$ & $2.46 \pm 0.80$ & this paper \\
78P/Gehrels 2 & $525-590$ & $1.88 \pm 0.75$ & this paper \\
& $525-775$ & $0.72 \pm 1.45$ & this paper \\
43P/Wolf-Harrington & $544-641$ & $0.34^{\star}$ & Lowry \& Fitzsimmons (2005) \\
46P/Wirtanen & $540-670$ & 8.3 & Lamy et al. (1998) \\
\hline COMETARY NUCLEI & & & \\
\hline 143P/Kushida-Muramatsu & $544-641$ & $3.75^{\star}$ & Lowry \& Fitzsimmons (2005) \\
133P/Elst-Pizarro & $544-641$ & $-1.29^{\star}$ & Lowry \& Fitzsimmons (2005) \\
1P/Halley & visual range & $6 \pm 3$ & Thomas \& Keller (1989) \\
10P/Tempel 2 & visual range & $20 \pm 3$ & Jewitt \& Luu (1989) \\
49P/Arend-Rigaux & visual range & -2.1 & Fitzsimmons et al. (1994) \\
82P/Gehrels 3 & visual range & $13.3 \pm 0.1$ & De Sanctis et al. (2000) \\
28P/Neujimin 1 & visual range & $10.3 \pm 3.0$ & Campins et al. (2007) \\
162P/Siding Spring & visual range & $9.2 \pm 1.0$ & Campins et al. (2007) \\
\hline
\end{tabular}

Notes. ${ }^{(a)}$ Values marked with ${ }^{\star}$ have been derived by us using measured Af $\rho$ data in Eq. (4).

Figure 11 displays the normalised reflectivities of the comae (in the innermost $1.4 \times 10^{4} \mathrm{~km}$ ) measured for all the target comets. All the comae appear to be redder than the Sun, which is generally considered a rule for cometary dust (Jewitt \& Meech 1986).

The colour of the dust coma can also be computed in terms of the reddening (see e.g. Lara et al. 2003), which indicates the percentage of change in the strength of the continuum per $1000 \AA$ (even if, when close to the Sun, we cannot exclude the broadband filters being contaminated by strong gas emission bands, especially in the $V$ filter). This parameter is given by

reddening $=\frac{1}{A f \rho(\lambda)} \frac{A f \rho\left(\lambda^{\prime}\right)-A f \rho(\lambda)}{\lambda^{\prime}-\lambda}$

where $\lambda$ and $\lambda^{\prime}$ are the central wavelengths of the filters. It is evident that this parameter is strongly dependent on the spectral range and the observational parameters selected for the analysis, therefore a straightforward comparison between values measured by different observers and for other targets (e.g., cometary nuclei) is not obvious. Table 7 summarises the values for our target comets in different spectral ranges (always obtained with photometry in aperture of radius $7.2 \times 10^{3} \mathrm{~km}$ ) compared with other values for comae and nuclei found in the literature ${ }^{3}$. Values for 32P/Comas Solá, 56P/Slaughter-Burnham and 78P/Gehrels 2 in the range $525-590 \mathrm{~nm}$ seam well in agreement with values computed in a similar spectral range (544-641 nm), using in Eq. (4) the values for $V$ and $R$ A $f \rho$ of 1 coma and 2 nuclei (upper limits) by Lowry \& Fitzsimmons (2005). If we further extend our spectral range $(525-775 \mathrm{~nm})$, the agreement is maintained, but unfortunately it is difficult to compare our measured values with results obtained in different spectral ranges from photometric and/or spectral observation of other nuclei (Lamy et al. 2009; De Sanctis et al. 2000; Campins et al. 2007; Jewitt \& Luu 1989; Fitzsimmons et al. 1994; Thomas \& Keller 1989). As a general consideration, at least among the SPC family cometary comae tend to be redder than the Sun, but bluer than bare nuclei.

\footnotetext{
${ }_{3}$ It may be that not all the available values have been listed in the Table, but we are confident that the two extremes (49P/Arend-Rigaux and 10P/Tempel 2) bracket all the measurements in the visible range.
}

\section{Dust mass production rate}

The Afo parameter is considered a good proxy for the dust activity, but it does not directly provide the dust production rate $Q_{\mathrm{d}}$. To obtain a first-order quantitative estimate of the dust mass-loss rate for the target comets, we decided to apply a "photometric model", which relates the optical photometry to the dust production rate via some realistic assumptions on the cometary dust environment. The method is derived from the one used by Jewitt (2009) to compute the dust production rate of active Centaurs in the region between 5 and $12 \mathrm{AU}$, and was already successfully applied to estimating the dust production rate of the Oort comet C/2007 D1 (LINEAR) (Mazzotta Epifani et al. 2010).

The method consists of computing the apparent magnitude of the pure coma $m_{\mathrm{d}}$ in an annulus between an inner $\phi$ and a conveniently chosen outer $\phi^{\prime}$ as

$$
m_{\mathrm{d}}=-2.5 \log \left(10^{-0.4 R}-10^{-0.4 R^{\prime}}\right)
$$

where $R$ and $R^{\prime}$ are the magnitudes in filter $R$ within apertures of radii $\phi$ and $\phi^{\prime}$, respectively. The values of $\phi=3.48^{\prime \prime}$ and $\phi^{\prime}=6.38^{\prime \prime}$ were adopted to be sure both to sample only the dust coma and exclude any possible nucleus contribution $(\phi=$ $3.48^{\prime \prime}$ ) and to minimise the effects of sky background, remaining within the "end" of the apparent visible coma $\left(\phi^{\prime}=6.38^{\prime \prime}\right)$. The photometry is then used to estimate the total cross-section of the coma dust particles in the projected annulus, using the inverse square law expressed by Russell (1916) as

$p_{R} \Phi(\alpha) C_{\mathrm{d}}=2.25 \times 10^{22} \pi r^{2} \Delta^{2} 10^{-0.4\left(M_{\mathrm{d}}-m_{\mathrm{S}}\right)}$

where $r$ and $\Delta$ are the heliocentric and geocentric distances expressed in $\mathrm{AU}, \alpha$ the comet phase angle, and $m_{\mathrm{S}}$ the apparent magnitude of the Sun in the $R$ filter. For the geometric albedo $p_{R}$, we decided to adopt the classical value of 0.04 (commonly assumed for the nucleus of short period comets). The quantity $\Phi(\alpha)=10^{-0.4 \alpha \beta}$ represents the phase darkening: for the phase coefficient $\beta$, we decided to adopt the value $\beta=0.045 \mathrm{mag} /{ }^{\circ}$, which is the average of the few measured phase coefficient values for short period comets (values for 8 comets in the range 0.025-0.06 mag/ ${ }^{\circ}$, see Lamy et al. 2004).

The dust cross-section $C_{\mathrm{d}}$ derived from the photometry, obtained by combining Eqs. (5) and (6), is related to the dust mass 
Table 8. Summary of grain velocity obtained from the application of the inverse numerical model, for several comets at the heliocentric distances where our target comets have been observed.

\begin{tabular}{|c|c|c|c|c|}
\hline Comet & $\overline{\text { Ref. }}$ & $\begin{array}{l}\text { Reference grain size } \\
{[\mathrm{cm}]}\end{array}$ & $\begin{array}{c}\text { Heliocentric distance }^{a} \\
{[\mathrm{AU}]}\end{array}$ & $\begin{array}{c}\text { Grain velocity } \\
{[\mathrm{m} / \mathrm{s}]}\end{array}$ \\
\hline \multirow{3}{*}{ 6P/d'Arrest } & (1) & 0.1 & 2.02 & 10 \\
\hline & & & 2.44 & $\ldots$ \\
\hline & & & 2.65 & $\ldots$ \\
\hline \multirow[t]{3}{*}{ 46P/Wirtanen } & (2) & 0.01 & 2.02 & 23 \\
\hline & & & 2.44 & 15 \\
\hline & & & 2.65 & 13 \\
\hline \multirow[t]{3}{*}{$65 \mathrm{P} / \mathrm{Gunn}$} & (2) & 0.01 & 2.02 & $\ldots$ \\
\hline & & & 2.44 & $\ldots$ \\
\hline & & & 2.65 & 24 \\
\hline \multirow{3}{*}{ 67P/Churyumov-Gerasimenko } & (3) & 1 & 2.02 & $9^{I}, 2^{\circ}$ \\
\hline & & & 2.44 & $\ldots$ \\
\hline & & & 2.65 & $\ldots$ \\
\hline \multirow{3}{*}{ 103P/Hartley 2} & (4) & 0.1 & 2.02 & 4 \\
\hline & & & 2.44 & 3 \\
\hline & & & 2.65 & 3 \\
\hline
\end{tabular}

References. (1): Fulle (1990); (2) Colangeli et al. (1998); (3) Fulle et al. (2004); (4) Epifani et al. (2001).

Notes. ${ }^{(a)} r=2.02 \mathrm{AU}$ refers to our 78P/Gehrels 2 observation. $r=2.44 \mathrm{AU}$ refers to our $32 \mathrm{P} / \mathrm{Comas}$ Solá observation. $r=2.65$ AU refers to our $56 \mathrm{P} /$ Slaughter-Burnham observation. ${ }^{(b)}$ I refers to preperihelion, $\mathrm{O}$ to postperihelion.

$M_{\mathrm{d}}$ through the adoption of a power-law dust size distribution. In this case, the total mass between a minimum $\left(a_{-}\right)$and a maximum $\left(a_{+}\right)$particle radius is

$M_{\mathrm{d}}=\frac{4}{3} \pi \Gamma \int_{a_{-}}^{a_{+}} a^{3-q} \mathrm{~d} a$.

Several studies based on fits of short period comet tails by an inverse numerical model (Fulle 1987, 1989) show that the size distribution power index can vary between -3.2 for comet 103P/Hartley 2 (Epifani et al. 2001) and 2P/Encke (Epifani et al. 2001) and -4.0 for comet 65P/Gunn (Colangeli et al. 1998), for grains between $a_{-}=10 \mu \mathrm{m}$ and $a_{+}=1 \mathrm{~cm}$ (see a summary in Fulle 1999). We then decided to adopt the average value of computed size distribution power index $q=-3.5$ in the above size range. Therefore, Eq. (7) turns into the expression for the total mass:

$M_{\mathrm{d}}=\frac{4}{3} \rho\left(a_{-} a_{+}\right)^{1 / 2} C_{\mathrm{d}}$

where $\rho=1000 \mathrm{~kg} / \mathrm{m}^{3}$ is the commonly assumed bulk density for compact cometary grains. The size of the average dust grain scatterer is $\langle a\rangle=\left(a_{-} a_{+}\right)^{1 / 2} \sim 30 \mu \mathrm{m}$, consistent with the observation that the dust coma of target comets is reddish compared to the Sun (see Sect. 3.4).

The dust-loss rate is then derived by considering the time of residence $\tau(r)$ of dust grains in the (projected) annulus between $\phi$ and $\phi^{\prime}$ (expressed in radians) equal to

$\tau(r)=\frac{1.5 \times 10^{11} \Delta\left(\phi^{\prime}-\phi\right)}{v(r)}$

where $v(r)$ is the radial outflow speed of the dust grains from the nucleus at the heliocentric distance $r$ of observation. The issue on $v(r)$ is critical, since the dust velocity is strictly dependent on dust size. Small grains $(a \sim 1 \mu \mathrm{m})$ in an active comet are expected to be dynamically well coupled to the gas and should have terminal velocity comparable to gas flow speed. At $r \sim 2 \mathrm{AU}$, the thermal velocity for the gas, appropriate to a temperature of $185 \mathrm{~K}$, is $v_{\text {gas }}=438 \mathrm{~m} / \mathrm{s}$. On the contrary, larger grains can be accelerated only to a lower terminal velocity than the gas flow speed, depending on many factors (dust-to-gas ratio by mass, nucleus radius, active fraction of nucleus surface, mass of gas, gas-production rate, etc. See Newburn \& Spinrad 1985; Singh et al. 1992, for detailed descriptions of the theory). For example, a simplification given by Probstein's theory (Probstein 1969; Fulle 1990) predicts a dust velocity close to $10 \%$ of the gas value for spherical dust grains emitted from a perfectly homogeneous nucleus. In the past, the application of the inverse numerical model to comet images allowed reconstruction of the dynamical evolution of the dust environment for a number of comets, including the dust grain velocity (in most cases for grains up to $1 \mathrm{~cm}$ ) at several heliocentric distances. Table 8 summarises the results for several comets at the heliocentric distances where our target comets have been observed: the grains appear to be much slower than the expanding gas, and also much slower than foreseen by the Bobrovnikoff relation (Bobrovnikoff 1954) $v_{\text {grain }}=v_{\text {bob }} \sim\left(\frac{\mu}{\mu_{\mathrm{H}_{2} \mathrm{O}}}\right) r^{-0.5}=600 r^{-0.5}$ for heliocentric distances $r \leq 3 \mathrm{AU}$, where the cometary activity is mostly driven by the sublimation of water ice and therefore $\mu$ (atomic weight of the sublimating gas) is that of water. Therefore we decided to compute a range of dust mass loss rate for each target comet, corresponding to dust grain velocity between 1 and an estimated realistic upper limit of $25 \mathrm{~m} / \mathrm{s}$. Table 9 summarises, for each comet, the values derived for all the above defined quantities and the obtained range of the dust production rate $Q_{\mathrm{d}}$. Figure 12 summarises the published dust mass loss rate for short period comets (as per April 2010), and allows the comparison of the model dust mass loss rate obtained in present paper for comets 32P/Comas Solá, 56P/Slaughter-Burnham and 78P/Gehrels 2 among the values available up to now for the family.

A direct comparison is not straightforward, since several model and observative factors highly influence the resulting dust mass loss rate; e.g., our results are given in terms of a lowerto-upper limit, based on realistic assumption on the grain velocity, which remains unknown at present (together with its dependence on grain size). Nevertheless, it is possible to observe that the general trend is, as expected, toward a decrease in the dust production rate with the increase in the heliocentric distance, corresponding to a decrease in the efficiency of the 
Table 9. Model dust mass loss-rate for target comets.

\begin{tabular}{|c|c|c|c|c|c|}
\hline \multirow[t]{2}{*}{ Comet } & \multirow[t]{2}{*}{$m_{\mathrm{d}}{ }^{a}$} & \multirow[t]{2}{*}{$C_{\mathrm{d}}^{b}\left[\mathrm{~m}^{2}\right]$} & \multirow[t]{2}{*}{$M_{\mathrm{d}}^{c}$} & \multicolumn{2}{|c|}{$Q_{\mathrm{d}}^{d}[\mathrm{~kg} / \mathrm{s}]$} \\
\hline & & & & Lower limit & Upper limit \\
\hline 32P/Comas Solá & 16.682 & $1.42 \times 10^{8}$ & $5.98 \times 10^{6}$ & 2 & 46 \\
\hline 56P/Slaughter-Burnham ${ }^{e}$ & 17.330 & $8.22 \times 10^{7}$ & $3.46 \times 10^{6}$ & 1 & 24 \\
\hline 78P/Gehrels 2 & 13.950 & $7.91 \times 10^{8}$ & $3.34 \times 10^{7}$ & 14 & 345 \\
\hline
\end{tabular}

Notes. ${ }^{(a)}$ Coma magnitude in the projected annulus between $\phi=3.48^{\prime \prime}$ and $\phi^{\prime}=6.38^{\prime \prime}$ (see text). ${ }^{(b)}$ Derived dust cross-section . ${ }^{(c)}$ Derived dust mass in the coma annulus . ${ }^{(d)}$ Derived dust mass loss rate: the lower limit corresponds to grains crossing the projected coma annulus at $v=1 \mathrm{~m} / \mathrm{s}$, the upper limit corresponds to grains crossing the projected coma annulus at $v=25 \mathrm{~m} / \mathrm{s}$. ${ }^{(e)}$ Average values between the two observing nights.

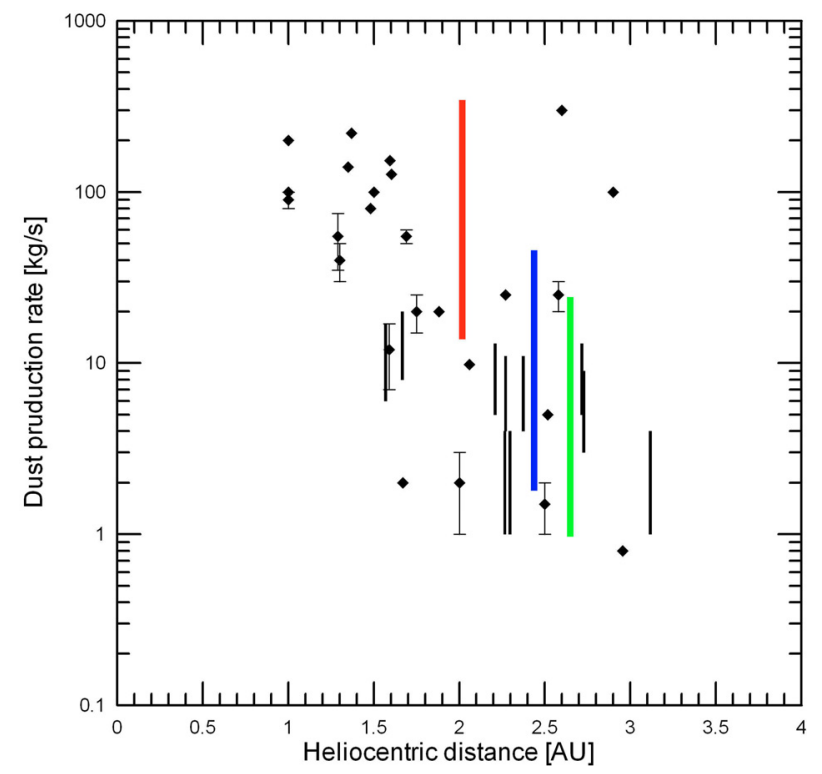

Fig. 12. Dust mass loss rate for short period comets. The black symbols are for single measurements, the black thin vertical lines correspond to a range assigned to the comet, depending on its classification: "typical" or "depleted", based on their abundances in carbonchain species (following the classification by A'Hearn et al. 1985). Values have been found in literature for comets 2P/Encke (Epifani et al. 2001), 4P/Faye (Lamy et al. 2009), 10P/Tempel 2 (Lamy et al. 2009), 17P/Holmes (Lamy et al. 2009), 21P/Grigg-Skjellerup (Fulle et al. 1993), 37P/Forbes (Lamy et al. 2009), 37PForbes (Newburn \& Spinrad 1985), 44P/Reinmuth 2 (Lamy et al. 2009), 46P/Wirtanen (Colangeli et al. 1998), 50P/Arend (Lamy et al. 2009), 59P/KearnsKwee (Lamy et al. 2009), 59P/Kearns-Kwee (Newburn \& Spinrad 1985), 63P/Wild 1 (Lamy et al. 2009), 63P/Wild 1 (Colangeli et al. 1998), 67P/Churyumov-Gerasimenko (Hanner et al. 1985; Fulle et al. 2004), 71P/Clark (Lamy et al. 2009), 84P/Giclas (Lamy et al. 2009), 103P/Hartley 2 (Epifani et al. 2001), 106P/Shuster (Lamy et al. 2009), 112P/Urata Nijima (Lamy et al. 2009), 114P/Wiseman-Skiff (Lamy et al. 2009). The thick vertical lines are for the model dust mass loss rate of comets presented in this paper, giving ranges for a grain velocity between 1 and $25 \mathrm{~m} / \mathrm{s}$. Thick blue line: 32P/Comas Solá; thick green line: 56P/Slaughter-Burnham; thick red line: 78P/Gerhels 2.

$\mathrm{H}_{2} \mathrm{O}$-ice sublimation process. As a remarkable exception, comet 63P/Wild 1, observed between $r_{\mathrm{h}}=2.5$ and 3 AU (Colangeli et al. 1998), appears quite active in this framework, with a dust production rate $Q_{\mathrm{d}}$ at $r_{\mathrm{h}} \sim 3 \mathrm{AU}(\sim 100 \mathrm{~kg} / \mathrm{s})$ comparable with the general cometary trend much closer to the Sun.

Among the comets characterised in the present paper, 32P/Comas Solá and 56P/Slaughter-Burnham seem to act as standard "dust emitter" $\left(Q_{\mathrm{d}}\right.$ from 2 to $46 \mathrm{~kg} / \mathrm{s}$ at $r_{\mathrm{h}}=2.4 \mathrm{AU}$ and $Q_{\mathrm{d}}$ from 1 to $24 \mathrm{~kg} / \mathrm{s}$ at $r_{\mathrm{h}}=2.65 \mathrm{AU}$, respectively), while comet $78 \mathrm{P} /$ Gerhels $\left(Q_{\mathrm{d}}\right.$ from 14 to $345 \mathrm{~kg} / \mathrm{s}$ at $\left.r_{\mathrm{h}}=2.0 \mathrm{AU}\right)$ appears to be more active than the average at that heliocentric distance. Snapshot observations are not perfectly suitable to characterising the "dust emitting" level of a comet, and instead can suggest the need to carefully monitor the target along its orbital branch and for more than one perihelion passage. This will help in the characterisation of the short period comets as major suppliers of the inner interplanetary dust cloud (Sykes et al. 2004; LevasseurRegourd et al. 2007), and in refining the time scale of the surviving of a body in a cometary form, before its activity is chunked off by the formation of a crust or mantle (possibly "dormant" comets, Whitman et al. 2006) or completely exhausted (Carvano et al. 2008; Licandro et al. 2008).

\section{Summary and conclusions}

We performed multicolour broad-band photometry of the short period comets 32P/Comas Solá, 56P/Slaughter-Burnham and $78 \mathrm{P} /$ Gehrels 2, observed when close to their perihelion from the $1.52 \mathrm{mt}$ Loiano telescope (Italy) during two observing nights in October 2004. Our aims were i), to characterise their dust coma morphology; ii), to derive coma profiles and A $f \rho$ parameter, so as to investigate the steadiness of the dust coma; iii), to characterise the dust coma colour; and iv), to model their dust production rate, on the basis of realistic assumptions on dust grain size and velocity.

Our main results can be summarised as follows:

1. The dust coma morphology of all comets has been investigated by means of several techniques, namely the adaptive Laplace filtering method and the radial renormalisation method, in order to enhance even faint dust structures and detect subtle deviations and variations from the mean brightness. 32P/Comas Solá did not show any dust feature, and both 56P/Slaughter-Burnham and 78P/Gehrels 2 deviated from a "normal" dust coma. 56P/Slaughter-Burnham showed a north/south asymmetry in the dust distribution, persisting over the two observing nights. 78P/Gehrels 2 presented two nearly axisymmetric lobes centred on the comet-Sun line, probably a rotationally generated feature.

2. For comet $78 \mathrm{P} / \mathrm{Gehrels} 2$, it was possible to derive the $1-\mathrm{D}$ surface brightness profile with a fitted slope of $m=-1.54 \pm$ 0.05 , which is just above the limit of a steady-state coma (with dominant distortion effect by solar radiation pressure). Our data on this comet do not allow to distinguish between a steady-state and a non-steady-state dust emission.

3 . For all the comets, the $R$-band $\mathrm{A} f \rho$ was computed in an aperture of radius $\rho=7.3 \times 10^{3} \mathrm{~km}$ (to minimise effects of the sky background): for comet 32P/Comas Solá, it was $127 \pm 10 \mathrm{~cm}$; for comet $56 \mathrm{P} /$ Slaughter-Burnham, it was $109 \pm 12 \mathrm{~cm}$ on 5 October and $97 \pm 10 \mathrm{~cm}$ on 6 October (weighted average between the two observing nights: $102 \pm 8 \mathrm{~cm}$ ); for comet 
$78 \mathrm{P} /$ Gehrels 2,846 $\pm 55 \mathrm{~cm}$. When regarded in the framework of the short period comet family, these results confirm the general trend of A $f \rho$ decreasing (as the dust production rate) with increasing $r_{\mathrm{h}}$, and for comet 32P/Comas Solá the variation, even if in two different perihelion passages, is very coherent.

4. The analysis of the coma colours revealed, for all the targets, a dust redder than the Sun (which is generally considered standard for cometary dust).

5. The "photometric model" applied to the dust coma allowed estimation of the dust production rate for all the targets, under realistic assumptions on grain velocity (as derived from measured and modelled values found in literature for comets of the same class) and size (as confirmed by dust coma colours measured during the observing run for the targets). Comets 32P/Comas Solá and 56P/Slaughter-Burnham are quite "standard" comets, with $Q_{\mathrm{d}}$ from 2 to $46 \mathrm{~kg} / \mathrm{s}$ at $r_{\mathrm{h}}=2.4 \mathrm{AU}$ and $Q_{\mathrm{d}}$ from 1 to $24 \mathrm{~kg} / \mathrm{s}$ at $r_{\mathrm{h}}=2.65 \mathrm{AU}$, respectively. Comet 78P/Gehrels 2 is a quite "dusty" comet ( $Q_{\mathrm{d}}$ from 14 to $345 \mathrm{~kg} / \mathrm{s}$ at $r_{\mathrm{h}}=2.0 \mathrm{AU}$ ), as it is more active than the average in the family at that heliocentric distance.

These results give a picture of two "standard" comets (32P/Comas Solá and 56P/Slaughter-Burnham) and a very "dusty" target (78P/Gehrels 2).

Snapshot observations, such as those presented in this paper, are useful to characterise the general characteristics of single targets (e.g., dust coma morphology and colours), but they should also be considered a "starting point" for a complete characterisation of the more interesting targets in the whole family complex, especially for the dust production rate. The evolution of this parameter should be carefully monitored over the whole orbital arc, and possibly for more than one perihelion passage, in order to investigate the contribution of the major "dust emitter" short-period comets to the inner interplanetary dust cloud complex and to pose more stringent constraints to the models describing the evolution of a minor body into its possible "dormant" or "extinct" phase and the interrelations between different families of bodies in the inner Solar System.

Acknowledgements. We gratefully acknowledge funding from Italian Space Agency (ASI) under contract I/015/07/0.

\section{References}

A'Hearn, M. F., Schleicher, D. G., Feldman, P. D., Millis, R. L., \& Thompson, D. T. 1984, AJ, 89, 579

A’Hearn, M. F., Millis, R. L., Schleicher, D. G., Osip, D. J., \& Birch, P. V. 1985 , Icarus, 118, 223

Benest, D., \& Gonczi, R. 1992, in LPI, Asteroids, Comets and Meteors 1991, 69 Birkle, K., \& Boehnhardt, H. 1992, EM\&P, 57, 191

Boehnhardt, H., \& Birkle, K. 1994, A\&ASS, 107, 101

Bobrobnikoff, N. T. 1954, AJ, 59, 356

Brownlee, D. E., Tsou, P., Aléon, J., et al. 2006, Science, 314, 1711

Campins, H., Licandro, J., Pinilla-Alonso, N., et al. 2007, AJ, 134, 1626

Cartwright, I. M., Fitzsimmons, A., Williams, I. P., \& Kemp, S. N. 1997, PSS, $45,7,821$

Carvano, J. M., Ferraz-Mello, S., \& Lazzaro, D. 2008, A\&A, 489, 2,811
Colangeli, L., Bussoletti, E., Cecchi Pestellini, C., et al. 1998, Icarus 134, 35 De Sanctis, M. C., Lazzarin, M., Barucci, M. A., Capria, M. T., \& Coradini, A. 2000, A\&A, 354, 1086

Epifani, E., Rotundi, A., Foster, M. J., et al. 1999, PSS, 47, 765

Epifani, E., Colangeli, L., Fulle, M., et al. 2001, Icarus, 149, 339

Farnham, T. L. 2009, PSS, 57, 1192

Fink, U. 1994, ApJ, 423, 462

Fink, U., Fevig, R. A., Tegler, S. C., \& Romanishin, W. 1997, PSS, 45, 11, 1383

Fitzsimmons, A., Dahlgren, M., Lagerkvist, C.-I., Magnusson, P., \& Williams, I. P. 1994, A\&A, 282, 634

Fulle, M. 1987, A\&A, 171, 327

Fulle, M. 1989, A\&A, 217, 283

Fulle, M. 1990, A\&A, 230, 220

Fulle, M. 1999, PSS, 47, 827

Fulle, M., Mennella, V., Rotundi, A., et al. 1993, A\&A, 276, 582

Fulle, M., Barbieri, C., Cremonese, G., et al. 2004, A\&A, 422, 357

Gehrz, R. D., \& Ney, E. P. 1992, Icarus, 100, 162

Hanner, M. S., Tedesco, E., Tokunaga, A. T., et al. 1985, Icarus, 64, 11

Holmberg, J., Flynn, C., \& Portinari, L. 2006, MNRAS, 367, 449

Królikowska, M., Sitarski, G., \& Szutowicz, S. 1998, A\&A, 335, 757

Królikowska, M., Sitarski, G., \& Szutowicz, S. 2001, A\&A, 368, 676

Jewitt, D. C. 2009, AJ, 137, 4296

Jewitt, D. C., \& Luu, J. X. 1989, AJ, 97, 1766

Jewitt, D. C., \& Meech, K. J. 1986, AJ, 310, 937

Jewitt, D. C., \& Meech, K. J. 1987, ApJ, 317, 992

Lamy, P. L., Toth, I., Jorda, L., \& Weaver, H. A. 1988, A\&A, 335, L25

Lamy, P. L., Toth, I., Gruen, E., et al. 1996, Icarus, 201, 674

Lamy, P. L., Toth, I., Fernández, Y. R., \& Weaver, H. A. 2004, in Comets II, ed. M., Festou, H. U., Keller, \& H. A., Weaver (Tucson: Univ. Arizona Press)

Lamy, P. L., Toth, I., Weaver, H. A., A'Hearn, M. F., \& Jorda, L. 2009, A\&A, 508,1045

Landolt, A. U. 1992, AJ, 104, 1, 340

Lara, L.-M., Licandro, J., Oscoz, A., \& Motta, V. 2003, A\&A, 497, 843

Lara, L.-M., Rodrigo, R., Tozzi, G. P., Boehnhardt, H., \& Leisy, P. 2004a, A\&A, 420,371

Lara, L.-M., Tozzi, G. P., Boehnhardt, H., Di Martino, M., \& Schulz, R. 2004b, A\&A, 422, 717

Lara, L.-M., Gutierrez, P. J., De Leon, J., \& Licandro, J. 2005, EM\&P, 97, 165

Lara, L.-M., Licandro, J., \& Tozzi, G. P. 2009, A\&A, 497, 843

Levasseur-Regourd, A. C., Mukai, T., Lasue, J., \& Okada, Y. 2007, PSS, 55, 9, 1010

Licandro, J., Alvarez-Candal, A., de Léon, J., et al. 2008, A\&A, 483, 861

Lowry, S. C., \& Fitzsimmons, A. 2005, MNRAS, 358, 641

Lowry, S. C., \& Weissman, P. R. 2003, Icarus, 164, 492

Lowry, S. C., Fitzsimmon, A., Cartwright, I. M., \& Williams, I. P. 1999, A\&A, 349,649

Lowry, S. C., Fitzsimmons, A., \& Collander-Brown, S. 2003, A\&A, 397, 329

Mazzotta Epifani, E., Palumbo, P., Capria, M. T., et al. 2008, MNRAS 390, 265

Mazzotta Epifani, E., Dall'Ora, M., Di Fabrizio, L., et al. 2010, A\&A, 513, A33

Meech, K. J., Hainaut, O. R., \& Marsden, B. G. 2004, Icarus, 170, 463

Newburn, R. L., \& Spinrad, H. 1985, AJ, 90, 12, 2591

Probstein, R. F. 1969, in Problems of Hydrodynamics and continuum mechanics, ed. F., Bishopp, et al. (Philadelphia: Soc. Indust. Appl. Math), 568

Russell, H. N. 1916, ApJ, 43, 173

Schulz, R. 2002, in Proceedings of Asteroids, Comets, Meteors (ACM 2002), ESA-SP-500

Schulz, R., Stuewe, J. A., Boehnhardt, H., Gaessler, W., \& Tozzi, G. P. 2003, A\&A, 398, 345

Schulz, R., Stuewe, J. A., \& Boehnhardt, H. 2004, A\&A, 422, L19

Sekanina, Z. 1993, AJ, 105, 702

Singh, P. D., de Almeida, A. A., \& Huebner, W. F. 1992, AJ, 104, 2, 848

Snodgrass, C., Fitzsimmons, A., \& Lowry, S. C. 2008, MNRAS, 385, 737

Sykes, M. V., Gruen, E., Reach, W. T., \& Jenniskens, P. 2004, in Comets II, ed.

M., Festou, H. H., Keller, \& H. A., Weaver (Tucson: Univ. Arizona Press)

Thomas, N., \& Keller, H. U. 1989, A\&A, 213, 487

Tozzi, G. P., Boehnhardt, H., \& Lo Curto, G. 2003, A\&, 398, L41

Whitman, K., Morbidelli, A., \& Jedicke, R. 2006, Icarus, 183, 1, 101 